\title{
Electron spin relaxation in bilayer graphene
}

\author{
L. Wang and M. W. Wv \\ Hefei National Laboratory for Physical Sciences at Microscale and Department of Physics, \\ University of Science and Technology of China, Hefei, Anhui, 230026, China
}

(Dated: November 24, 2018)

\begin{abstract}
Electron spin relaxation due to the D'yakonov-Perel' mechanism is investigated in bilayer graphene with only the lowest conduction band being relevant. The spin-orbit coupling is constructed from the symmetry group analysis with the parameters obtained by fitting to the numerical calculation according to the latest report by Konschuh et al. [Phys. Rev. B 85, 115423 (2012)] from first principles. In contrast to single-layer graphene, the leading term of the out-of-plane component of the spin-orbit coupling in bilayer graphene shows a Zeeman-like term with opposite effective magnetic fields in the two valleys. This Zeeman-like term opens a spin relaxation channel in the presence of intervalley scattering. It is shown that the intervalley electron-phonon scattering, which has not been reported in the previous literature, strongly suppresses the in-plane spin relaxation time at high temperature whereas the intervalley short-range scattering plays an important role in the in-plane spin relaxation especially at low temperature. A marked nonmonotonic dependence of the in-plane spin relaxation time on temperature with a minimum of several hundred picoseconds is predicted in the absence of the short-range scatterers. This minimum is comparable to the experimental data. Moreover, a peak in the electron density dependence of the in-plane spin relaxation time at low temperature, which is very different from the one in semiconductors, is predicted. We also find a rapid decrease in the in-plane spin relaxation time with increasing initial spin polarization at low temperature, which is opposite to the situation in both semiconductors and single-layer graphene. A strong anisotropy between the out-of- and in-plane spin relaxations at high temperature is also revealed with the out-of-plane spin relaxation time being about two orders of magnitude larger than the in-plane one. Detailed comparisons of the temperature and electron density dependences of the spin relaxation with the existing experiments [Phys. Rev. Lett. 107, 047206 (2011), Phys. Rev. Lett. 107, 047207 (2011) and Nano Lett. 11, 2363 (2011)] are reported.
\end{abstract}

PACS numbers: 72.25.Rb, 81.05.ue, 71.10.-w, 71.70.Ej

\section{INTRODUCTION}

In recent years, single-layer graphene has received much attention due to its two dimensionality, Diraclike energy spectrum and outstanding spin-coherence properties $\stackrel{-14}{-14}$ Specifically, the hyperfine interaction and spin-orbit coupling (SOC) are considerably weak,,$\frac{15-25}{2}$ suggesting a long spin relaxation time (SRT) in singlelayer graphene. This long SRT makes single-layer graphene a promising candidate for spintronic application. So far, a number of experiments on spin relaxation have been carried out, with the SRTs being orders of magnitude shorter than theoretical prediction $\underline{3.26-41}$ This pronounced discrepancy has provoked many investigations on the extrinsic effects of spin relaxation such as adatoms, $\stackrel{24.42-47}{\underline{47}}$ curvature $\stackrel{16,48-50}{\underline{\underline{n}}}$ substrate effects $44,51,52$ and contacts $30,39,40,53$ Consisting of two layer graphene, bilayer graphene (BLG) also possesses good spin-coherence properties $20,21,54-57$ Additionally, in contrast to single-layer graphene, BLG has a tunable gap caused by an out-of-plane electric field $, 55,56,58-62$ which can be used to turn on or off the electronic transport. In addition, in the absence of space inversion symmetry, the intrinsic SOC in the pseudospin space of BLG provides an out-of-plane effective magnetic field near the Dirac points whereas the intrinsic SOC of single-layer graphene only induces a shift of the energy spectrum $36,44,55$ Moreover, the electron-phonon coupling becomes more complex in BLG due to the existence of more phonon modes $\underline{63-65}$ So far, some efforts have been made to investigate the intravalley electron-phonon coupling. $\underline{-65}$ Specifically, the intravalley electron-acoustic (AC) phonon coupling has been calculated by Viljas and Heikkilä 63 with the continuum theory and also by Borysenko et al $\underline{\underline{64}}$ from first principles. To explore the intravalley electron-optical (OP) phonon coupling, the tight-binding model, density functional theory and first principles approach are employed by Viljas and Heikkilä, $\underline{63}$ Cappelluti et al $\underline{\underline{65}}$ and Borysenko et al.,$\frac{64}{}$ respectively. However, the intervalley electron-phonon coupling, which has been shown to play an important role in spin relaxation in rippled singlelayer graphene, $\stackrel{50}{\underline{5}}$ has not been reported in the previous literature. All these intriguing features make BLG an attractive material for investigation.

Very recently, some attention has been devoted to the spin relaxation in $\mathrm{BLG}, \underline{37-39,66-68}$ Experimentally, the SRTs of BLG on $\mathrm{SiO}_{2}$ substrate $\frac{37,38,66}{3}$ or in freely suspended BLG 68 are reported to be of the order of 0.01 1 ns. Both Yang et al ${ }^{66}$ and Han and Kawakami ${ }^{37}$ measured the temperature and carrier dependences of the SRT. Yang et al. ${ }^{66}$ showed that the SRT is weakly dependent on temperature. However, Han and Kawakami ${ }^{37}$ reported a marked decrease of the SRT with the increase of the temperature. As for the carrier density depen- 
dence, the decrease of the SRT with increasing carrier density at low temperature is reported in both experiments. However, at room temperature, Yang et al. ${ }^{66}$ observed an increase of the SRT with increasing density whereas the SRT measured by Han and Kawakami ${ }^{37}$ exhibits a marginal density dependence. In addition, Yang et al $\stackrel{66}{=}$ also showed that the SRT scales inversely with the mobility at both room and low temperatures. In contrast to the above two experiments based on the micromechanically exfoliated BLG samples, Avsar et al $l^{38}$ carried out the experiment in chemical vapor deposition grown BLG. The carrier density and temperature dependences of the SRT measured are comparable to the ones by Yang et $a l \underline{66}$ both quantitatively and qualitatively. Motivated by these experiments, Diez and Burkard 67 calculated the electron spin relaxation due to the D'yakonov-Perel' (DP) mechanism ${ }^{69}$ with only the long-range electronimpurity scattering included in BLG. They claimed to obtain a good agreement with the experimental data of Yang et al. ${ }^{66}$ It is noted that the effective SOC (referred to as the spin-orbit field in Ref. 67) used in their calculation is induced by an interlayer bias voltage together with the intrinsic SOC in the pseudospin space ${ }^{54}$ However, the intrinsic SOC in the pseudospin space becomes incomplete in the presence of the interlayer bias since the interlayer bias breaks the space inversion symmetry and leads to additional extrinsic SOC terms in the pseudospin space $\stackrel{55}{\underline{5}}$ Also even with only the intrinsic SOC in the pseudospin space in their calculation, they overlooked the out-of-plane component of the effective SOC. As will be shown later, the leading term of this component leads to a Zeeman-like term in the two valleys and makes a dominant contribution to the in-plane spin relaxation. Moreover, they neglected the electronphonon and electron-electron Coulomb scatterings. In fact, the electron-electron Coulomb scattering has been demonstrated to be very important for the spin relaxation in semiconductor systems ${ }^{70}-\underline{77}$ and also single-layer graphene at high temperature ${ }^{36}$ The electron-phonon scattering has also been shown to play an important role in spin relaxation in both semiconductors ${ }^{70}$ and singlelayer graphene ${ }^{36}$ at high temperature. Therefore, both have to be included in studying the spin relaxation.

In the present work, with the electron-electron Coulomb, (both the intra- and inter-valley) electronphonon, possible short-range ${ }^{\underline{9}}$ as well as long-range electron-impurity scatterings explicitly included, we investigate the electron spin relaxation due to the DP mechanism in BLG with only the lowest conduction band being relevant by the kinetic spin Bloch equation (KSBE) approach ${ }^{70}$ The SOC of the lowest conduction band near the Dirac points $\boldsymbol{\Omega}^{\mu}(\mathbf{k})$ is constructed from the symmetry group $C_{3}$ in the presence of an out-of-plane electric field $\stackrel{78}{=}$ Its three components $\Omega_{i}^{\mu}(\mathbf{k})(i=x, y, z)$ can be written as (see Appendix A)

$$
\begin{aligned}
\Omega_{x}^{\mu}(\mathbf{k})= & \alpha_{1}(k) \sin \theta_{\mathbf{k}}+\mu\left[\alpha_{2}(k) \sin 2 \theta_{\mathbf{k}}\right. \\
& \left.+\alpha_{3}(k) \sin 4 \theta_{\mathbf{k}}\right], \\
\Omega_{y}^{\mu}(\mathbf{k})= & -\alpha_{1}(k) \cos \theta_{\mathbf{k}}+\mu\left[\alpha_{2}(k) \cos 2 \theta_{\mathbf{k}}\right. \\
& \left.-\alpha_{3}(k) \cos 4 \theta_{\mathbf{k}}\right], \\
\Omega_{z}^{\mu}(\mathbf{k})= & \mu \beta_{1}(k)+\beta_{2}(k) \cos 3 \theta_{\mathbf{k}},
\end{aligned}
$$

with $\mu=1(-1)$ standing for $\mathrm{K}\left(\mathrm{K}^{\prime}\right)$ valleys and $k$ and $\theta_{\mathbf{k}}$ representing the magnitude and the polar angle of the momentum $\mathbf{k}$ relative to $\mathrm{K}\left(\mathrm{K}^{\prime}\right)$ points, respectively. The coefficients $\alpha_{i}(k)(i=1-3)$ and $\beta_{1,2}(k)$ are given in Appendix $\AA$ by fitting to the numerical results calculated according to the latest report by Konschuh et al ${ }^{55}$ from first principles with both the intrinsic and extrinsic SOC terms in the pseudospin space included. The out-of-plane component $\Omega_{z}^{\mu}(\mathbf{k})$ [Eq. (3)], which has not been reported in the previous literature, is induced by the intrinsic SOC in the pseudospin space in the absence of space inversion symmetry, whereas the in-plane component is contributed by both the intrinsic and extrinsic SOCs in the pseudospin space. This is very different from the case in single-layer graphene where the intrinsic SOC in the pseudospin space only induces a shift of the energy spectrum in the conduction band $36,44,55$ We find that the magnitudes of both the out-of- and inplane components decrease with the increase of $k$ at large momentum, indicating a suppression of the inhomogeneous broadening $\frac{71}{71}$ by increasing momentum. $\frac{55}{2}$ This is very different from the case in both semiconductors and

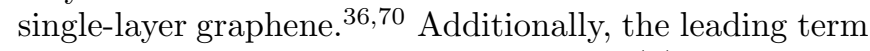
of the out-of-plane component, i.e., $\mu \beta_{1}(k)$, serves as a Zeeman-like term with opposite effective magnetic fields perpendicular to BLG plane in the two valleys, similar to the case in rippled single-layer graphene $\underline{50}$ This Zeemanlike term, together with the intervalley scattering, opens a spin relaxation channel. This spin relaxation channel suppresses the in-plane SRT significantly at high temperature by the intervalley electron-phonon scattering and also at low temperature by the short-range scattering. ${ }^{9}$ In addition, we find that although such Zeeman-like terms also exist in the in-plane components of the SOC, their contributions to the out-of-plane spin relaxation are marginal since these terms are of the high order of the momentum. This leads to a strong anisotropy between the in-plane and out-of-plane spin relaxations. We also find that the in-plane SRT shows a marked nonmonotonic dependence on temperature with a minimum down to several hundred picoseconds in the absence of short-range scatterers. This minimum is comparable to the experimental data. The nonmonotonic behavior results from the crossover between the weak and strong intervalley electron-phonon scattering. Moreover, we predict a peak in the density dependence at low temperature, which is very different from the one in semiconductors $\stackrel{77,79}{ }$ As for high temperature, the SRT shows a monotonic increase with increasing density. We also find that the in-plane SRT decreases rapidly with increasing initial spin polar- 
ization at low temperature, which is very different from the previous studies in both semiconductors $72,76,80,81$ and single-layer graphene ${ }^{36}$ Finally, we report detailed comparisons of the temperature and electron density dependences with the existing experiments $\frac{37,38,66}{3}$

This paper is organized as follows. In Sec. II, we present our model and construct the KSBEs. The main results are given in Sec. III. In Sec. IIIA, we investigate the temperature, electron density and also initial spin polarization dependences of the electron spin relaxation in the absence of short-range scatterers. The anisotropy of the spin relaxation is also addressed in this part. In Sec. IIIB, we show detailed comparisons of the temperature and electron density dependences of the spin relaxation with the existing experiments with the inclusion of short-range scatterers. We summarize in Sec. IV.

\section{MODEL AND KSBES}

We start our investigation from the AB-stacked BLG on $\mathrm{SiO}_{2}$ substrate. The spinless $\pi$-band structure can be described by the effective $4 \times 4$ Hamiltonian: $:^{55}$

$$
H_{\mathrm{TB}}(\tilde{\mathbf{k}})=\left(\begin{array}{cccc}
\Delta+V & \gamma_{0} f(\tilde{\mathbf{k}}) & \gamma_{4} f^{*}(\tilde{\mathbf{k}}) & \gamma_{1} \\
\gamma_{0} f^{*}(\tilde{\mathbf{k}}) & +V & \gamma_{3} f(\tilde{\mathbf{k}}) & \gamma_{4} f^{*}(\tilde{\mathbf{k}}) \\
\gamma_{4} f(\tilde{\mathbf{k}}) & \gamma_{3} f^{*}(\tilde{\mathbf{k}}) & -V & \gamma_{0} f(\tilde{\mathbf{k}}) \\
\gamma_{1} & \gamma_{4} f(\tilde{\mathbf{k}}) & \gamma_{0} f^{*}(\tilde{\mathbf{k}}) & \Delta-V
\end{array}\right)
$$

in the on-site orbital Bloch basis $\Psi_{\mathrm{A}_{1}}(\tilde{\mathbf{k}}), \Psi_{\mathrm{B}_{1}}(\tilde{\mathbf{k}}), \Psi_{\mathrm{A}_{2}}(\tilde{\mathbf{k}})$ and $\Psi_{\mathrm{B}_{2}}(\tilde{\mathbf{k}})$, where $\mathrm{A}_{1}$ refers to the A sublattice in the lower layer, $\mathrm{B}_{2}$ to the $\mathrm{B}$ sublattice in the upper layer, etc., and $\tilde{\mathbf{k}}$ represents the two-dimensional wave vector counted from the $\Gamma$ point. Here, $\gamma_{0}$ and $\gamma_{1}$ describe the nearest-neighbor intralayer and interlayer hoppings whereas $\gamma_{3}$ and $\gamma_{4}$ stand for the indirect hoppings between two layers. $\Delta$ represents the asymmetry in the energy shift of the on-site energy due to the interlayer hopping. The potential $V=e E_{z} d_{\text {eff }} / 2$ with $e, E_{z}$ and $d_{\text {eff }}$ representing the electron charge $(e>0)$, the out-of-plane electric field and the effective electrostatic bilayer distance, respectively. $f(\tilde{\mathbf{k}})=$ $e^{i \frac{a}{\sqrt{3}} \tilde{k}_{y}}\left[1+2 e^{-i \frac{\sqrt{3} a}{2} \tilde{k}_{y}} \cos \left(\frac{a}{2} \tilde{k}_{x}\right)\right]$ is the nearest-neighbor structural function of the graphene hexagonal lattice with $a$ denoting the lattice constant. Near the Dirac points, to the first order of the momentum $\mathbf{k}$ originated from the $\mathrm{K}\left(\mathrm{K}^{\prime}\right)$ points, $f(\tilde{\mathbf{k}}) \equiv p(\mathbf{k})=-\sqrt{3} a\left(\mu k_{x}-i k_{y}\right) / 2$. Then the effective $4 \times 4$ Hamiltonian near the Dirac points can be written as

$$
H^{\mu}(\mathbf{k})=\left(\begin{array}{cccc}
\Delta+V & \gamma_{0} p & \gamma_{4} p^{*} & \gamma_{1} \\
\gamma_{0} p^{*} & +V & \gamma_{3} p & \gamma_{4} p^{*} \\
\gamma_{4} p & \gamma_{3} p^{*} & -V & \gamma_{0} p \\
\gamma_{1} & \gamma_{4} p & \gamma_{0} p^{*} & \Delta-V
\end{array}\right)
$$

By exactly diagonalizing this Hamiltonian, one obtains the eigenvalue $\epsilon_{\mu \nu \mathbf{k}}$ and the eigenfunction $\psi_{\mathbf{k}}^{\mu \nu}$ with $\nu$ being the band index including two conduction bands and two valence ones. In our calculation, we concentrate on the heavily $n$-doped case with only the lowest conduction band being relevant whereas other bands are separated by large energy intervals. For convenience, the eigenvalue and the corresponding eigenfunction of the lowest conduction band at $\mathbf{k}$ are denoted by $\epsilon_{\mu \mathbf{k}}$ and $\psi_{\mathbf{k}}^{\mu}$, respectively.

By incorporating the spin degree of freedom, the effective Hamiltonian of the lowest conduction band is given by

$$
H_{\mathrm{eff}}^{\mu}=\epsilon_{\mu \mathbf{k}}+\boldsymbol{\Omega}^{\mu}(\mathbf{k}) \cdot \boldsymbol{\sigma} / 2 .
$$

Here, $\boldsymbol{\sigma}$ are the Pauli matrices. $\boldsymbol{\Omega}^{\mu}(\mathbf{k})$ stands for the effective magnetic field of the SOC, which is constructed from the symmetry analysis with the parameters obtained approximately by fitting to numerical results based on the report by Konschuh et al. .55 The analytical form of the SOC with three components $\Omega_{i}^{\mu}(\mathbf{k})$ $(i=x, y, z)$ is shown in Eqs. (113) and the explicit numerical calculation is given in Appendix A. It is noted that at large momentum, the analytical result agrees with the numerical one fairly well (see Appendix $\mathrm{A}$ ).

We then construct the microscopic KSBEs ${ }^{70}$ to study the electron spin relaxation in BLG. The KSBEs read ${ }^{70}$

$$
\partial_{t} \hat{\rho}_{\mu \mathbf{k}}=\left.\partial_{t} \hat{\rho}_{\mu \mathbf{k}}\right|_{\mathrm{coh}}+\left.\partial_{t} \hat{\rho}_{\mu \mathbf{k}}\right|_{\mathrm{scat}},
$$

where $\hat{\rho}_{\mu \mathbf{k}}$ represent the density matrices of electrons with the diagonal terms $\rho_{\mu \mathbf{k}, \sigma \sigma} \equiv f_{\mu \mathbf{k} \sigma}\left(\sigma= \pm \frac{1}{2}\right)$ describing the distribution functions and the off-diagonal ones $\rho_{\mu \mathbf{k},(1 / 2)(-1 / 2)}=\rho_{\mu \mathbf{k},(-1 / 2)(1 / 2)}^{*}$ denoting the spin coherence. The coherent term is given by

$$
\left.\partial_{t} \hat{\rho}_{\mu \mathbf{k}}\right|_{\mathrm{coh}}=-i\left[\boldsymbol{\Omega}^{\mu}(\mathbf{k}) \cdot \boldsymbol{\sigma} / 2+\hat{\Sigma}_{\mu \mathbf{k}}^{\mathrm{HF}}, \hat{\rho}_{\mu \mathbf{k}}\right],
$$

where $[A, B] \equiv A B-B A$ is the commutator; $\hat{\Sigma}_{\mu \mathbf{k}}^{\mathrm{HF}}=-\sum_{\mathbf{k}^{\prime}} V_{\mathbf{k}, \mathbf{k}^{\prime}}^{\mu} I_{\mathbf{k k}^{\prime}}^{\mu} \hat{\rho}_{\mu \mathbf{k}^{\prime}}$ represents the Coulomb Hartree-Fock (HF) term. ${ }^{72} V_{\mathbf{k}, \mathbf{k}^{\prime}}^{\mu}$ denotes the screened Coulomb potential with its form given in Appendix B. The form factor $I_{\mathbf{k k}^{\prime}}^{\mu}=\left|\psi_{\mathbf{k}}^{\mu \dagger} \psi_{\mathbf{k}^{\prime}}^{\mu}\right|^{2} .\left.\quad \partial_{t} \hat{\rho}_{\mu \mathbf{k}}\right|_{\text {scat }}$ are the scattering terms including the electronelectron Coulomb $\left(\left|V_{\mathbf{k}, \mathbf{k}^{\prime}}^{\mu}\right|^{2}\right)$, long-range electron-impurity $\left(\left|U_{\mathbf{k}, \mathbf{k}^{\prime}}^{\mu}\right|^{2}\right)$, intravalley electron-AC phonon $\left(\left|M_{\mu \mathbf{k}, \mu^{\prime} \mathbf{k}^{\prime}}^{\mathrm{AC}}\right|^{2}\right)$, electron-OP phonon $\left(\left|M_{\mu \mathbf{k}, \mu^{\prime} \mathbf{k}^{\prime}}^{\mathrm{OP}}\right|^{2}\right)$, electron-remoteinterfacial (RI) phonon $\left(\left|M_{\mu \mathbf{k}, \mu^{\prime} \mathbf{k}^{\prime}}^{\mathrm{RI}}\right|^{2}\right)$ and especially the intervalley electron-phonon scattering including

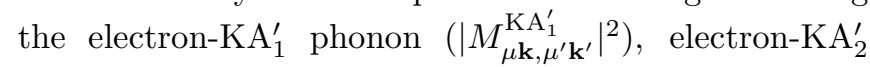
phonon $\left(\left|M_{\mu \mathbf{k}, \mu^{\prime} \mathbf{k}^{\prime}}^{\mathrm{KA}^{\prime}}\right|^{2}\right)$, electron-KE' phonon $\left(\left|M_{\mu \mathbf{k}, \mu^{\prime} \mathbf{k}^{\prime}}^{\mathrm{KE}^{\prime}}\right|^{2}\right)$ and electron-KZ phonon $\left(\left|M_{\mu \mathbf{k}, \mu^{\prime} \mathbf{k}^{\prime}}^{\mathrm{KZ}}\right|^{2}\right)$ scatterings. Their detailed expressions can be found in Ref. 36,82 Here, $\mathrm{KA}_{1}^{\prime}, \mathrm{KA}_{2}^{\prime}$ and $\mathrm{KE}^{\prime}$ are the in-plane phonon modes corresponding to the representations $A_{1}^{\prime}, A_{2}^{\prime}$ and $E^{\prime}$ of group $D_{3 h}$, respectively whereas KZ stands for the out-of-plane phonon mode ${ }^{64.83-85}$ It is noted that the intravalley electron-phonon scattering has been reported in the previous literature as mentioned in the introduction, $\underline{63,64}$ 
However, to the best of our knowledge, there is no specific report to the intervalley electron-phonon scattering. In this work, we derive the intervalley electron-phonon scattering matrix elements using the tight-binding model according to the arXiv version of Ref. 63. The scattering matrix elements are given by

$$
\begin{gathered}
\left|M_{\mu \mathbf{k}, \mu^{\prime} \mathbf{k}^{\prime}}^{\mathrm{KA}}\right|^{2}=\frac{3 \hbar^{2}}{4 \rho \Omega_{\mathrm{KA}_{1}^{\prime}}} \delta_{\mu^{\prime},-\mu}\left[\mid \psi_{\mathbf{k}}^{\mu \dagger}\left(2 \sqrt{3} \gamma_{0}^{\prime} \sigma_{\mathrm{D}}^{23}+a \gamma_{4}^{\prime} \gamma_{\mathrm{D}}^{0} / l_{4}\right)\right. \\
\left.\times\left.\psi_{\mathbf{k}^{\prime}}^{\mu^{\prime}}\right|^{2}+\left|\psi_{\mathbf{k}}^{\mu \dagger}\left(2 \sqrt{3} i \gamma_{0}^{\prime} \sigma_{\mathrm{D}}^{01}-a \gamma_{4}^{\prime} \gamma_{\mathrm{D}}^{3} \gamma_{\mathrm{D}}^{5} / l_{4}\right) \psi_{\mathbf{k}^{\prime}}^{\mu^{\prime}}\right|^{2}\right], \quad(9) \\
\left|M_{\mu \mathbf{k}, \mu^{\prime} \mathbf{k}^{\prime}}^{\mathrm{KA}}\right|^{\prime}=\frac{3 a^{2} \gamma_{4}^{\prime 2}}{2 \rho l_{4}^{2}} \frac{\hbar^{2}}{2 \Omega_{\mathrm{KA}_{2}^{\prime}}} \delta_{\mu^{\prime},-\mu}\left[\left|\psi_{\mathbf{k}}^{\mu \dagger} \gamma_{\mathrm{D}}^{3} \gamma_{\mathrm{D}}^{5} \psi_{\mathbf{k}^{\prime}}^{\mu^{\prime}}\right|^{2}\right. \\
\left.+\left|\psi_{\mathbf{k}}^{\mu \dagger} \gamma_{\mathrm{D}}^{0} \psi_{\mathbf{k}^{\prime}}^{\mu^{\prime}}\right|^{2}\right], \\
\left|M_{\mu \mathbf{k}, \mu^{\prime} \mathbf{k}^{\prime}}^{\mathrm{KE}}\right|^{2}=\frac{3 a^{2}}{\rho l_{4}^{2}} \frac{\hbar^{2}}{4 \Omega_{\mathrm{KE}^{\prime}}} \delta_{\mu^{\prime},-\mu}\left[\mid \psi_{\mathbf{k}}^{\mu \dagger}\left(\gamma_{3}^{\prime} \gamma_{\mathrm{D}}^{1} \gamma_{\mathrm{D}}^{5}-i \gamma_{3}^{\prime} \gamma_{\mathrm{D}}^{2}\right.\right. \\
\left.-\gamma_{4}^{\prime} \gamma_{\mathrm{D}}^{0}\right)\left.\psi_{\mathbf{k}^{\prime}}^{\mu^{\prime}}\right|^{2}+\left|\psi_{\mathbf{k}}^{\mu \dagger}\left(i \gamma_{3}^{\prime} \gamma_{\mathrm{D}}^{2}-\gamma_{3}^{\prime} \gamma_{\mathrm{D}}^{1} \gamma_{\mathrm{D}}^{5}-\gamma_{4}^{\prime} \gamma_{\mathrm{D}}^{0}\right) \psi_{\mathbf{k}^{\prime}}^{\mu^{\prime}}\right|^{2} \\
\left.+4\left|\psi_{\mathbf{k}}^{\mu \dagger} \gamma_{4}^{\prime} \gamma_{\mathrm{D}}^{3} \gamma_{\mathrm{D}}^{5} \psi_{\mathbf{k}^{\prime}}^{\mu^{\prime}}\right|^{2}\right], \\
\left|M_{\mu \mathbf{k}, \mu^{\prime} \mathbf{k}^{\prime}}^{\mathrm{KZ}}\right|^{2}=\frac{\gamma_{1}^{\prime 2}}{\rho} \frac{\hbar^{2}}{2 \Omega_{\mathrm{KZ}}} \delta_{\mu^{\prime},-\mu}\left|\psi_{\mathbf{k}}^{\mu \dagger}\left(\gamma_{\mathrm{D}}^{1} \gamma_{\mathrm{D}}^{5}+i \gamma_{\mathrm{D}}^{2}\right) \psi_{\mathbf{k}^{\prime}}^{\mu^{\prime}}\right|^{2},
\end{gathered}
$$

where $\rho$ is the mass density of the BLG; ${ }^{63} \Omega_{\mathrm{KA}_{1}^{\prime}}, \Omega_{\mathrm{KA}_{2}^{\prime}}$, $\Omega_{\mathrm{KE}^{\prime}}$ and $\Omega_{\mathrm{KZ}}$ are the energy spectra of $\mathrm{KA}_{1}^{\prime}, \mathrm{KA}_{2}^{\prime}$, $\mathrm{KE}^{\prime}$, and $\mathrm{KZ}$ phonon modes, respectively; ${ }^{64,83,84} \gamma_{i}^{\prime}(i=$ $0,1,3,4)$ is the derivative of $\gamma_{i}$ with respect to the corresponding hopping bond length;65 $l_{4}$ is the bond length of the interlayer hopping $\gamma_{4} ; \gamma_{\mathrm{D}}^{i}(i=0-3,5), \sigma_{\mathrm{D}}^{01}$ and $\sigma_{\mathrm{D}}^{23}$ are $4 \times 4$ Dirac matrices given in Appendix $\mathrm{C}^{86} \mathrm{We}$ will show that the above intervalley electron-phonon scattering plays a significant role in the in-plane spin relaxation in our investigation. The remaining scattering matrix elements such as $\left|V_{\mathbf{k}, \mathbf{k}^{\prime}}^{\mu}\right|^{2},\left|U_{\mathbf{k}, \mathbf{k}^{\prime}}^{\mu}\right|^{2}$, and $\left|M_{\mu \mathbf{k}, \mu^{\prime} \mathbf{k}^{\prime}}^{\lambda}\right|^{2}$ $(\lambda=\mathrm{AC}$, OP and RI) are given in Appendix [B] It is noted that we also include the short-range scattering in our calculation with the scattering term laid out later in this paper.

\section{NUMERICAL RESULTS}

By numerically solving the KSBEs, one obtains the time evolution of spin polarization along direction $\mathbf{n}$ $P(t)=\sum_{\mu \mathbf{k}} \operatorname{Tr}\left[\hat{\rho}_{\mu \mathbf{k}}(t) \boldsymbol{\sigma} \cdot \mathbf{n}\right] / N_{e}$ with $N_{e}$ being the electron density. Then the SRT can be determined from the slope of the envelope of the spin polarization $P(t) \stackrel{70}{ }$ The initial spin polarization $P(0)$ is set to be $2.5 \%$ and the spin-polarization direction $\mathbf{n}$ is along the $x$-axis unless otherwise specified. It is noted that all the parameters used in our calculation are listed in Table I. It is also noted that the SOC is calculated both fully numerically following the approach by Konschuh et al. ${ }^{55}$ and analytically by using Eqs. (1/3). The SRT calculated with the analytical form of the SOC and with the explicit numerical one agrees fairly well with each other.
TABLE I: Parameters used in the calculation (from Ref. 55 unless otherwise specified).

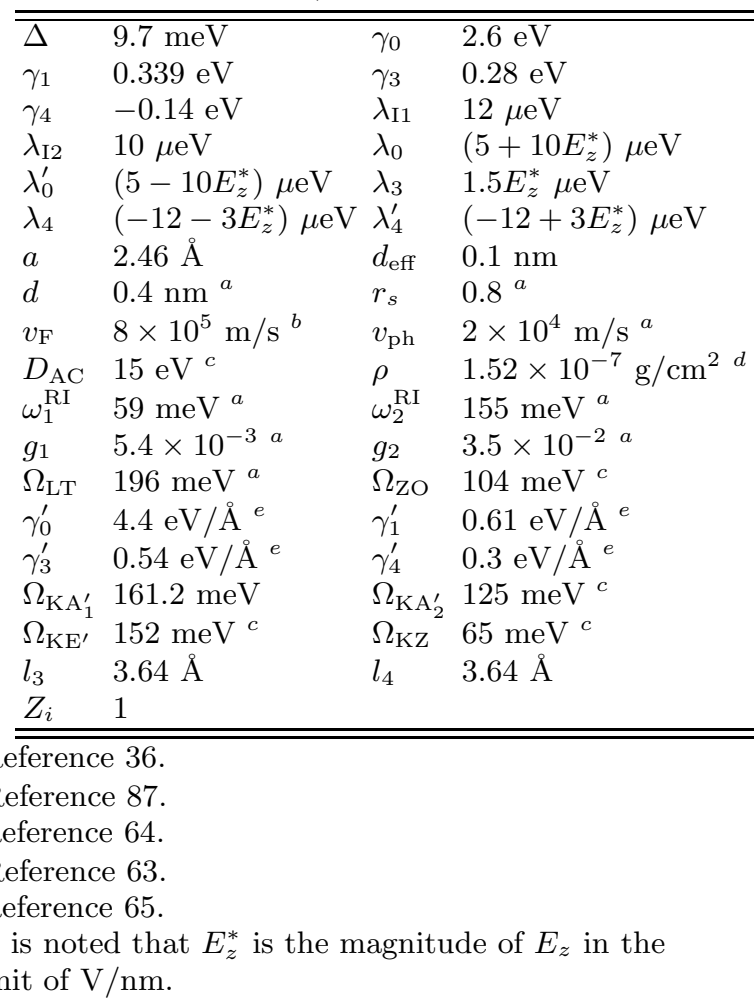

\section{A. Spin relaxation in BLG with high mobilities}

In our calculation, the out-of-plane electric field is chosen to be a typical value $E_{z}=0.14 \mathrm{~V} / \mathrm{nm}^{37}$ and the long-range impurity density is taken to be $N_{i}=$ $1.5 \times 10^{11} \mathrm{~cm}^{-2}$. The corresponding mobility in our investigation is of the order of $10^{4} \mathrm{~cm}^{2} /(\mathrm{V} \mathrm{s})$, which is about 1-2 orders of magnitude larger than those reported in the existing experiments in BLG on $\mathrm{SiO}_{2}$ substrate $37,38,66$ and comparable to the ones in the experiments in freely suspended BLG ${ }^{68}$ It is noted that the short-range scattering is not included in this subsection.

\section{Temperature dependence of spin relaxation}

We first investigate the temperature dependence of the spin relaxation. In Fig. 1] the SRT along the $x$-axis $\tau_{s_{x}}$ is plotted as function of temperature $T$. It is seen that $\tau_{s_{x}}$ changes little with the temperature at low temperature (i.e., $T \leq 60 \mathrm{~K}$ ). However, as $T$ becomes larger, the SRT decreases dramatically with increasing temperature. In particular, the SRT reaches down to several hundred picoseconds at $T=200 \mathrm{~K}$, which is comparable to the experimental values. $37,38,66,68$ A detailed comparison with the existing experiments will be discussed in Sec. IIIB. When $T$ further increases, the SRT begins to increase with $T$. 


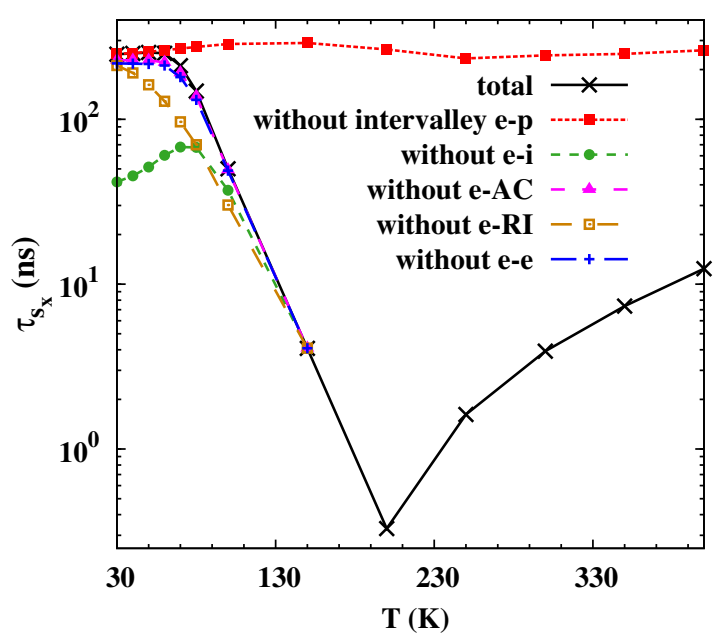

FIG. 1: (Color online) Total SRT along the $x$-axis $\tau_{s_{x}}(\times)$ and the calculation without the long-range electron-impurity $(\bullet)$, electron-AC-phonon $(\boldsymbol{\Lambda})$, electron-RI-phonon $(\square)$, electronelectron Coulomb $(+)$ or intervalley electron-phonon $(\boldsymbol{\square})$ scattering as function of temperature $T$. It is noted that when $T>150 \mathrm{~K}$, only the total SRT and the calculation without the intervalley electron-phonon scattering are shown since the remaining ones almost coincide with the total SRT. In the calculation, the electron density $N_{e}=3 \times 10^{12} \mathrm{~cm}^{-2}$.

To understand the behavior of the spin relaxation, we show in Fig. 1 the temperature dependence of the SRT calculated with the electron-electron Coulomb, electronRI-phonon, electron-AC-phonon, long-range electronimpurity, or intervalley electron-phonon scattering removed, separately. We find that at low temperature, the intervalley electron-phonon scattering is negligible. Therefore, spins relax independently in the two valleys and the SRT is solely determined by the intravalley spin relaxation channel. Specifically, the long-range electron-impurity scattering is dominant, which leads to a weak temperature dependence of the SRT $\underline{36,50}$ With the increase of the temperature, the contribution of the long-range electron-impurity scattering becomes marginal whereas the intervalley electron-phonon scattering becomes important. This intervalley scattering opens another spin relaxation channel together with the Zeeman-like term with opposite effective magnetic fields in the two valleys, which is similar to the case in rippled single-layer graphene $\stackrel{50}{\underline{5}}$ This Zeeman-like term is given by $\mu \beta_{1}(k)$ [Eq. (3)] approximately. Then the spin relaxation due to the above intervalley spin relaxation channel can be approximated by the rate equations ${ }^{50}$

$$
\dot{\mathbf{S}}_{\mu k}(t)+\mathbf{S}_{\mu k}(t) \times \boldsymbol{\omega}_{\mu k}+\frac{\mathbf{S}_{\mu k}(t)-\mathbf{S}_{-\mu k}(t)}{\tau_{v}(k)}=0,
$$

where $\mathbf{S}_{\mu k}, \boldsymbol{\omega}_{\mu k}=\left[\mu \beta_{1}(k)\right] \hat{\mathbf{z}}$ and $\tau_{v}(k)$ represent the inplane spin vectors in each valley, spin precession vector along the $z$-axis and the intervally electron-phonon scattering time, respectively. According to the report by Zhang et al.,$\frac{50}{5}$ the in-plane SRT is given by

$$
\tau_{s_{x, y}}(k)=\left\{\begin{array}{c}
\tau_{v}(k) \text { weak scattering } \\
\text { limit }\left(\left|\beta_{1}(k)\right| \tau_{v}(k) \geq 1\right) \\
\frac{2}{\left|\beta_{1}(k)\right|^{2} \tau_{v}(k)} \quad \text { strong scattering } \\
\text { limit }\left(\left|\beta_{1}(k)\right| \tau_{v}(k) \ll 1\right)
\end{array}\right.
$$

Here, $\tau_{s_{y}}$ stands for the SRT along the $y$-axis. Since the system we investigate is always in the degenerate regime, we take $\tau_{s_{x, y}}(k) \approx \tau_{s_{x, y}}\left(k_{F}\right)$ approximately with $k_{F}$ representing the Fermi wave vector. In our calculation, the SRT along the $x$-axis is solely determined by the intervalley electron-phonon scattering at $T \sim 100-400 \mathrm{~K}$. At $T=100 \mathrm{~K},\left|\beta_{1}\left(k_{F}\right)\right| \tau_{v}\left(k_{F}\right) \approx 160$, i.e., the intervalley electron-phonon scattering is in the weak scattering limit. As a result, the SRT $\tau_{s_{x}}=\tau_{v}\left(k_{F}\right)$ according to Eq. (14), which decreases with the enhancement of the intervalley scattering as the temperature increases. Nevertheless, at $T=400 \mathrm{~K},\left|\beta_{1}\left(k_{F}\right)\right| \tau_{v}\left(k_{F}\right) \approx 0.04$, i.e., the intervalley scattering is in the strong scattering limit. Then the SRT $\tau_{s_{x}}=2 /\left[\left|\beta_{1}\left(k_{F}\right)\right|^{2} \tau_{v}\left(k_{F}\right)\right]$ from Eq. (14), which increases with increasing of $T$. Therefore, the SRT first decreases then increases with increasing temperature when the intervalley scattering changes from the weak to strong scattering limit. The crossover between the weak and strong intervalley scattering limit is determined by $\tau_{v}\left(k_{F}\right)^{-1} \approx\left|\beta_{1}\left(k_{F}\right)\right| \underline{\underline{50}}$ At this crossover point,

$$
\tau_{s_{x}} \approx\left|\beta_{1}\left(k_{F}\right)\right|^{-1} \text {. }
$$

Here $\tau_{s_{x}} \approx 315 \mathrm{ps}$, very close to the value $330 \mathrm{ps}$ shown in the figure. It is noted that the electron-electron Coulomb scattering is unimportant in the whole temperature regime.

\section{Electron-density dependence of spin relaxation}

Then we turn to study the electron-density dependence. In Fig. 2, the SRT along the $x$-axis is plotted against the electron density at $T=100(300) \mathrm{K}$. One observes a peak in the electron-density dependence of the SRT at $T=100 \mathrm{~K}$ whereas the SRT increases with the increase of the electron density when $T=300 \mathrm{~K}$. We first focus on the case at $T=100 \mathrm{~K}$ and show the SRT with the intervalley electron-phonon scattering excluded in Fig. 2. It is seen that when the electron density is low, the intervalley electron-phonon scattering is marginal. The SRT is then determined by the intravalley spin relaxation channel and hence increases with the suppression of the inhomogeneous broadening ${ }^{71}$ as the electron density increases $\frac{55}{5}$ The suppression of the inhomogeneous broadening can be understood from the decrease of the spin splitting with increasing momentum at high momentum [see Eqs. (113)], as shown in Fig. 9 in Appendix A. This behavior is very different from the case in both semiconductors and single-layer graphene $3 \underline{36.70}$ When the electron density further increases, the intervalley electronphonon scattering becomes important. This intervalley 
scattering is always in the weak scattering limit with $\left|\beta_{1}\left(k_{F}\right)\right| \tau_{v}\left(k_{F}\right) \geq 40$ and therefore $\tau_{s_{x}}=\tau_{v}\left(k_{F}\right)$ according to Eq. (14). As a result, the SRT decreases with the increase of the intervalley scattering and hence the increase of the electron density $\underline{50}$ Therefore, in the whole electron-density regime, a peak is observed. It is noted that this peak is very different from the one predicted by Jiang and $\mathrm{Wu}^{79}$ in semiconductors where the peak is attributed to the crossover from the nondegenerateto-degenerate limit. As for the case at $T=300 \mathrm{~K}$, the SRT is always dominated by the intervalley electronphonon scattering as shown in Fig. 2 by comparing the SRT calculated with and without the intervalley electronphonon scattering. Since this intervalley scattering is in the strong scattering limit with $\left|\beta_{1}\left(k_{F}\right)\right| \tau_{v}\left(k_{F}\right) \leq 0.75$, the SRT $\tau_{s_{x}}=2 /\left[\left|\beta_{1}\left(k_{F}\right)\right|^{2} \tau_{v}\left(k_{F}\right)\right]$ from Eq. (14)). This SRT increases with increasing electron density since both $\left|\beta_{1}\left(k_{F}\right)\right|$ and the intervalley scattering time $\tau_{v}\left(k_{F}\right)$ decrease with the increase of the electron density 50,55

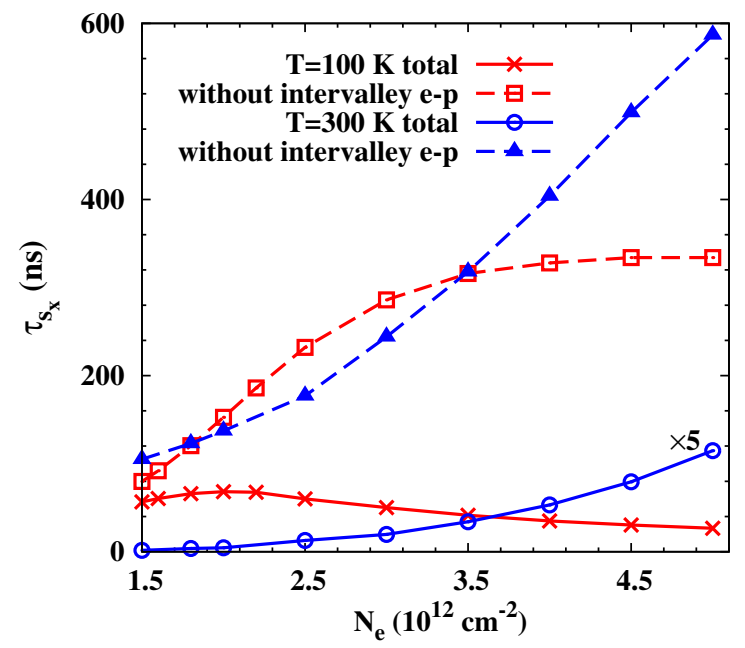

FIG. 2: (Color online) SRT along the $x$-axis $\tau_{s_{x}}$ as function of the electron density $N_{e}$ with $\times(\circ)$ and without $\square(\boldsymbol{\Delta})$ the intervalley electron-phonon scattering at $T=100$ (300) K.

\section{Influence of initial spin polarization}

The initial spin polarization dependences of the SRT along the $x$-axis at $T=100$ and $300 \mathrm{~K}$ are shown in Fig. 3. It is seen that the SRT decreases rapidly with the increase of initial spin polarization $P$ at $T=$ $100 \mathrm{~K}$ whereas the SRT shows a slight increase with increasing $P$ at $T=300 \mathrm{~K}$. The rapid decrease of the SRT at $T=100 \mathrm{~K}$ is very different from the previous studies in both semiconductors ${ }^{72,76,80,81}$ and single-layer graphene ${ }^{36}$ where the SRT increases significantly with increasing initial spin polarization. In the previous studies, the increase of the SRT originates from the contribution

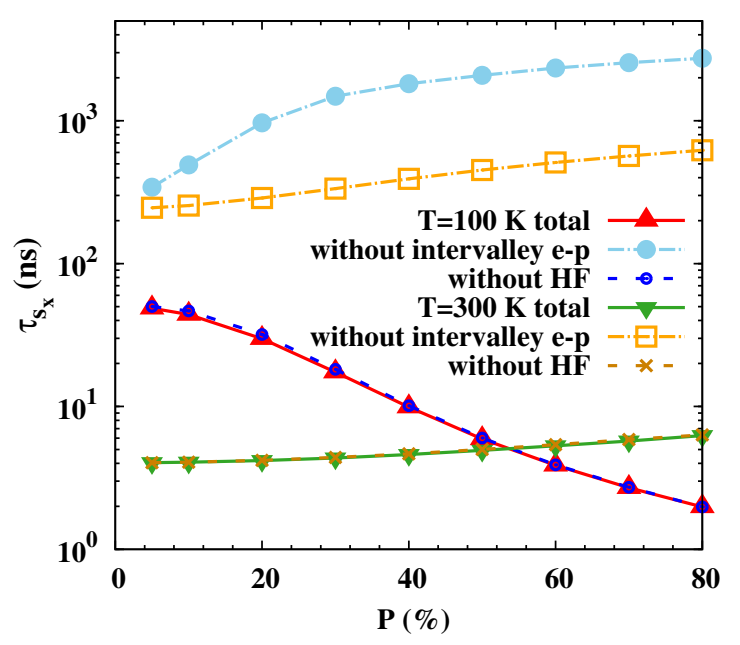

FIG. 3: (Color online) Total SRT along the $x$-axis $\tau_{s_{x}} \boldsymbol{\Delta}(\boldsymbol{\nabla})$ and the calculation without the Coulomb HF term $\circ(x)$ or the intervalley electron-phonon scattering $\bullet(\square)$ as function of the initial spin polarization $P$ at $T=100$ (300) K. In the calculation, the electron density $N_{e}=3 \times 10^{12} \mathrm{~cm}^{-2}$.

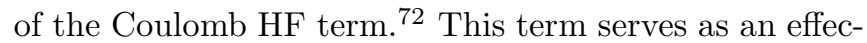
tive magnetic field along the direction of the spin polarization, which blocks the spin precession induced by the Dresselhaus ${ }^{88}$ and/or Rashba ${ }^{89}$ SOCs and slows down the spin relaxation. However, the contribution of the Coulomb HF term is marginal to the spin relaxation in our investigation, which is shown in Fig. 3 by comparing the the SRT calculated with and without the Coulomb HF term. Here, the SRT is determined by the intervalley electron-phonon scattering, as shown in Fig. 3 by comparing the calculation with and without the intervalley electron-phonon scattering. The intervalley electronphonon scattering transfers electrons between the two valleys. The transferred electrons experience opposite effective magnetic fields induced by the Zeeman-like term in the two valleys, which are not affected by the effective magnetic field induced by the Coulomb HF term. Moreover, the intervalley electron-phonon scattering is in the weak scattering limit at low temperature and hence the SRT $\tau_{s_{x}}=\tau_{v}\left(k_{F}\right)$ [see Eq. (14)]. The intervalley scattering time $\tau_{v}\left(k_{F}\right)$ decreases significantly with the increase of the initial spin polarization as the density of states increases with the polarization. Therefore, the SRT shows a rapid decrease with the initial spin polarization at $T=100 \mathrm{~K}$. As for the case of $T=300 \mathrm{~K}$, the SRT is also determined by the intervalley electron-phonon scattering in comparison with the case of $T=100 \mathrm{~K}$. However, in contrast to the case of $T=100 \mathrm{~K}$, the intervalley scattering is in the strong scattering limit. This makes the SRT present an opposite trend to the case of $T=100 \mathrm{~K}$. A mild increase of the SRT results from the insensitivity of $\tau_{v}\left(k_{F}\right)$ to the initial spin polarization at $T=300 \mathrm{~K}$. 


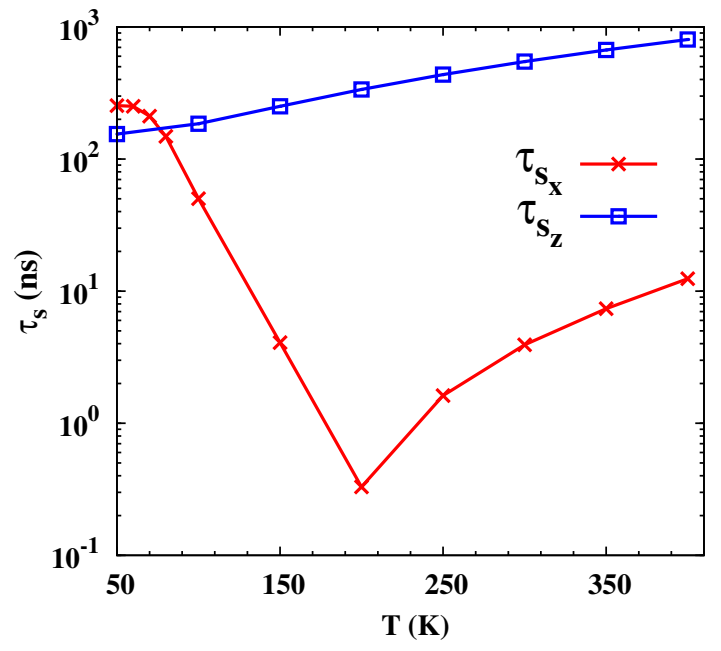

FIG. 4: (Color online) SRT along the $x(z)$-axis $\tau_{s_{x}}\left(\tau_{s_{z}}\right)$ as function of temperature $T$. In the calculation, the electron density $N_{e}=3 \times 10^{12} \mathrm{~cm}^{-2}$.

\section{Anisotropy of spin relaxation}

We also address the anisotropy of the spin relaxation with respect to the spin polarization direction. Due to the existence of the out-of-plane effective magnetic field [see Eq. (3)] ], the in-plane SRTs are identical $\stackrel{50}{=}$ For comparison, we plot the temperature dependence of the SRT along the $x(z)$-axis as the red curve with crosses (blue curve with open squares) in Fig. 4. It is seen that in comparison with the SRT along the $z$-axis, the one along the $x$-axis is comparable at low temperature and orders of magnitude smaller at high temperature. As mentioned previously, the SRT along the $x$-axis is strongly suppressed by the intervalley spin relaxation channel induced by the intervalley electron-phonon scattering and the Zeeman-like term in the two valleys at high temperature. However, the contribution of this spin relaxation channel to the SRT along the $z$-axis is marginal [see Eqs. (1-2)]. Therefore, a strong anisotropy between the out-of- and in-plane spin relaxations is observed at high temperature.

\section{B. Comparison with experiments}

As mentioned in the introduction, experiments on spin relaxation have been carried out in BLG on $\mathrm{SiO}_{2}$ substrate $\frac{37,38,66}{6}$ or in freely suspended BLG $\frac{68}{6}$ very recently by different groups. The in-plane SRTs in these experiments are of the order of 0.01-1 ns, which are comparable to our theoretical results at high temperature (several hundred picoseconds). This motivates us to carry out a detailed comparison with the existing experiments. In these experiments, the gate voltage, tem-
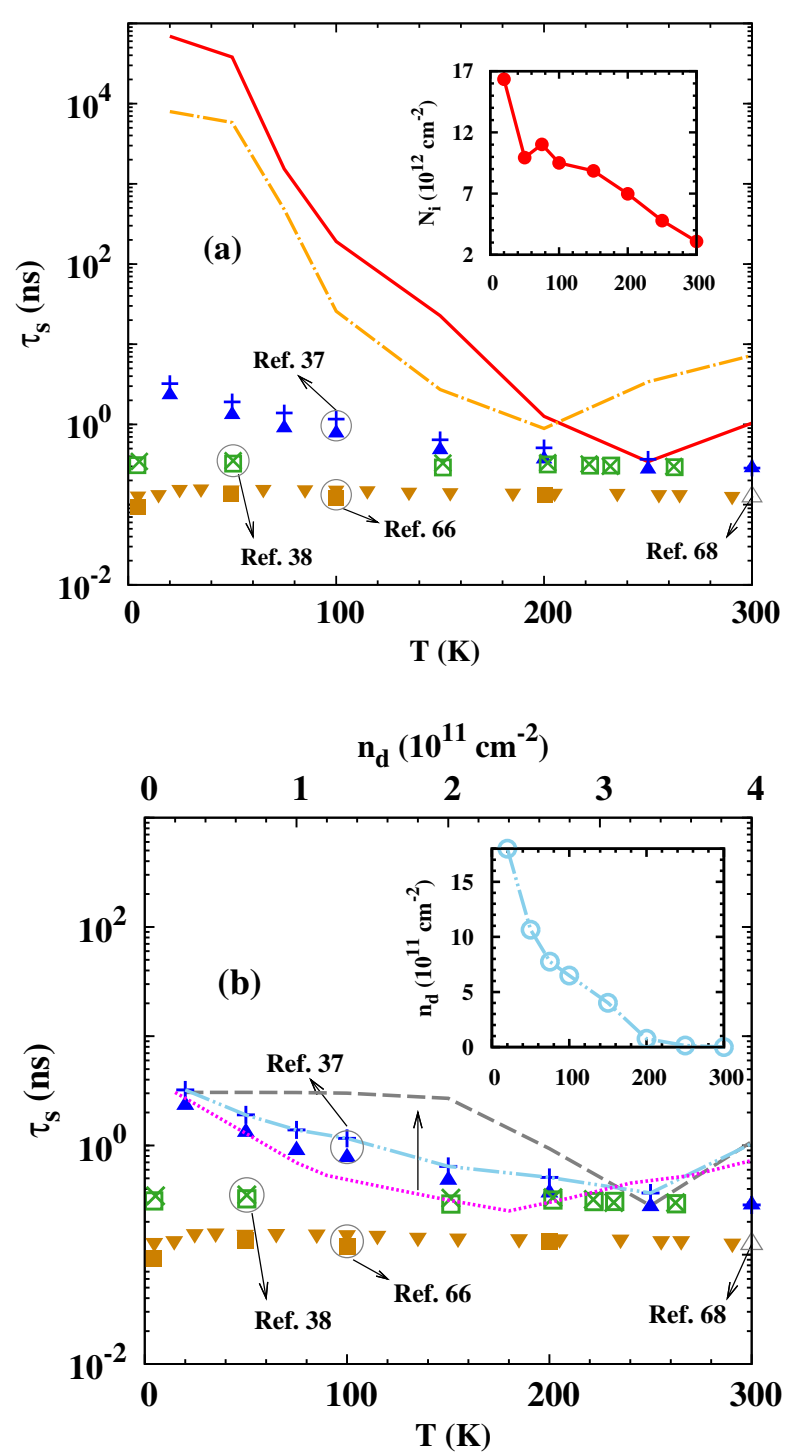

FIG. 5: (Color online) In-plane SRT $\tau_{s}$ as function of temperature $T .+(\mathbf{\Delta})$ corresponds to the case of the gate voltage $\mathrm{V}_{\mathrm{CNP}}+20$ (60) $\mathrm{V}$ in the experiment of Han and Kawakami, $37 \times(\square)$ stands for the case of electron density $N_{e}=0.75(1.5) \times 10^{12} \mathrm{~cm}^{-2}$ in the experiment of Avsar et $a l . ; \frac{38}{\nabla}(\mathbf{\square})$ represents the case of $N_{e}=0.7(2.2) \times 10^{12} \mathrm{~cm}^{-2}$ in the experiment of Yang et al. ${ }^{66} \Delta$ denotes the case of $N_{e}=10^{11} \mathrm{~cm}^{-2}$ in the experiment of Neumann et al. ${ }^{68}$ (a) Red solid (orange chain) curve stands for our calculation corresponding to the case of $\mathrm{V}_{\mathrm{CNP}}+20$ (60) $\mathrm{V}$ in the absence of the short-range scatterers. In the inset, we show the temperature dependence of the long-range impurity density $N_{i}$ corresponding to the case of $\mathrm{V}_{\mathrm{CNP}}+20 \mathrm{~V}$. (b) Grey dashed and skyblue double-dotted chain curves are obtained from our calculations corresponding to the case of $\mathrm{V}_{\mathrm{CNP}}+20 \mathrm{~V}$ by including the short-range scatterers, with a fixed short-range impurity density $n_{d}=2 \times 10^{10} \mathrm{~cm}^{-2}$ and a tunable $n_{d}$ (shown in the inset), respectively. We also show the dependence of $\tau_{s}$ on $n_{d}$ (with the scale on the top of the frame) at $T=50 \mathrm{~K}$ with other conditions the same as the case of $\mathrm{V}_{\mathrm{CNP}}+20 \mathrm{~V}$. The short-range potential strength $V_{0}=6 \times 10^{-17} \mathrm{meV} \mathrm{m}^{2}$. 
perature and electron density 90 (tuned by the gate voltage) are given explicitly. However, some parameters such as the out-of-plane electric field and the impurity density are not available in the experiments. In our calculation, the out-of-plane electric field is taken to be the ratio of the gate voltage to the thickness of the substrate approximately $\stackrel{16.46}{ }$ The impurity density is obtained by fitting to the mobility or the spin diffusion coefficient given in the experiments $\stackrel{91}{\underline{1}}$ In addition, we assume the initial spin polarization along the $x$-axis without loss of generality since the in-plane spin relaxations are identical as pointed out previously.

We first compare the temperature dependence of the SRTs obtained from the experiments and calculation from our model. In these experiments, the mobilities are reported of the order of $10^{2}-10^{4} \mathrm{~cm}^{2} /(\mathrm{Vs})$. The SRTs from different groups are comparable as shown in Fig. 5. However, the SRT from the experiment of Han and Kawakami ${ }^{37}$ decreases with increasing temperature whereas the ones from the experiments of Yang et $a l l^{66}$ and Avsar et al. $\frac{38}{6}$ show a marginal temperature dependence. In addition, Han and Kawakami ${ }^{37}$ reported the longest SRT among these experiments, indicating that their experimental data may contain less extrinsic effects. Motivated by this, we show our comparison with the experiment of Han and Kawakami 37 in Fig. 5(a). The experimental data with the gate voltage $\mathrm{V}_{\mathrm{CNP}}+20(60) \mathrm{V}$ corresponding to the electron density $N_{e}=1.47(4.4) \times 10^{12} \mathrm{~cm}^{-2}$, is labeled as $+(\mathbf{\Lambda})$. By fitting to the spin diffusion coefficient given in the experiment, we obtain the long-range impurity density $N_{i}$ shown in the inset as the curve with •. In contrast to the long-range impurity scattering, the contribution of other scatterings to the spin diffusion coefficient (mobility) is marginal. Then we calculate the SRT corresponding to $\mathrm{V}_{\mathrm{CNP}}+20(60) \mathrm{V}$ shown as the red solid (orange chain) curve. A crossover is observed in the temperature dependence of the SRT, similar to the one shown in Sec. IIIA. It is seen that the SRT from our calculation is comparable to the experimental data at high temperature. This suggests that the intervalley spin relaxation channel induced by the intervalley electron-phonon scattering and the Zeeman-like term in the two valleys addressed in Sec. IIIA, plays an important role at high temperature. Nevertheless, the SRTs are still larger than the experimental data with an observable difference at high temperature. Additionally, at low temperature, our results are orders of magnitude larger than the experimental ones since the intervalley electron-phonon scattering is marginal at low temperature and the SRT is determined by the long-range electron-impurity scattering in our calculation. Due to the existence of all these discrepancies, one has to take into account extrinsic effects such as adatoms,,$\underline{24,42}-\underline{47}$ curvature,,$\underline{16,48}-\underline{-50}$ substrate effects $44,51,52$ and also contacts: $30,39,40,53$ In the above experiments on BLG, the spin polarized electrons are all injected from ferromagnetic contacts $\underline{37,38,66,68}$ As reported, the ferromagnetic electrodes generate stray fields, which cause spin precession around the contact $\stackrel{53}{=}$ As a result, the stray fields may have a strong influence on the observed experimental results. Additionally, electron scattering by the tunneling contacts may also be responsible for the available experimental data $\underline{30,39,40}$

In addition to the above extrinsic effects, the shortrange scatterers may also be an extrinsic source. ${ }^{9,92,93}$ Here, we discuss the contribution of short-range scatterers 9 in detail. The scattering term induced by the short-range scatterers is given by

$$
\begin{aligned}
\left.\partial_{t} \hat{\rho}_{\mu \mathbf{k}}\right|_{\mathrm{SR}}= & -\pi n_{d} V_{0}^{2} \sum_{\mu^{\prime} \mathbf{k}^{\prime}}\left|\psi_{\mathbf{k}}^{\mu \dagger} \psi_{\mathbf{k}^{\prime}}^{\mu^{\prime}}\right|^{2} \delta\left(\epsilon_{\mu^{\prime} \mathbf{k}^{\prime}}-\epsilon_{\mu \mathbf{k}}\right) \\
& \times\left(\hat{\rho}_{\mu \mathbf{k}}-\hat{\rho}_{\mu^{\prime} \mathbf{k}^{\prime}}\right)+\text { H.c. }
\end{aligned}
$$

with $n_{d}$ and $V_{0}$ denoting the short-range impurity density and the constant short-range potential strength, respectively $\underline{\underline{9}}$ This scattering term contributes to both the intra- and inter-valley scatterings. The intervalley scattering opens an intervalley spin relaxation channel together with the Zeeman-like term in the two valleys [see Eq. (3)], similar to the intervalley electron-phonon scattering. However, in contrast to the intervalley electronphonon scattering, the intervalley scattering induced by the short-range scatterers is insensitive to the temperature and may play an important role in the in-plane spin relaxation especially at low temperature. Here, we choose a fixed short-range impurity density $n_{d}=2 \times 10^{10} \mathrm{~cm}^{-2}$ and potential strength $V_{0}=6 \times 10^{-17} \mathrm{meV} \mathrm{m}^{2}$. It is noted that although with this additional short-range scattering included, the mobility is still dominated by the longrange electron-impurity scattering since the contribution of the long-range electron-impurity scattering is about four orders of magnitude larger than that of the shortrange one. We calculate the temperature dependence of the SRT corresponding to the case of $\mathrm{V}_{\mathrm{CNP}}+20 \mathrm{~V}$ in the experiment of Han and Kawakami ${ }^{37}$ by including the additional short-range scattering. The result is plotted as the grey dashed curve in Fig. 5(b). A crossover is also observed at high temperature due to the contribution of the intervalley electron-phonon scattering as pointed out previously. By comparing our calculation with the experimental data labeled as + , we find that our result becomes comparable at low temperature. Furthermore, by fitting to the experimental data with tunable short-range impurity density shown as the curve with $\circ$ in the inset, our result labeled as the skyblue double-dotted chain curve agrees fairly well with the experimental data in the temperature regime lower than the crossover. As for the temperature higher than the crossover, our result becomes even larger than the experimental one once the short-range scattering is included. This is because that the intervalley electron-phonon scattering is in the strong scattering limit. With the inclusion of the short-range scattering, the intervalley scattering becomes stronger and the SRT becomes larger. This discrepancy may be attributed to other extrinsic effects.

As mentioned above, the SRT can be tuned by the short-range impurity density. We show the short-range 
impurity density dependence of the in-plane SRT at $T=50 \mathrm{~K}$ as the purple dotted curve in Fig. 5(b), with all other conditions remaining the same as the case of $\mathrm{V}_{\mathrm{CNP}}+20 \mathrm{~V}$. A valley is also observed, similar to the case of the temperature dependence discussed above. At low temperature, the intervalley electron-phonon scattering is negligible. The SRT is then determined by the intervalley short-range scattering. The valley originates from the crossover between the weak and strong intervalley short-range scattering limit by tuning the shortrange impurity density. At the crossover point, the SRT $\tau_{s} \approx 232 \mathrm{ps}$ according to Eq. (15), close to the value $252 \mathrm{ps}$ shown in the figure. This crossover point gives the minimum of the SRT and can be taken as a criterion for determining the possible contribution of the short-range scattering. Specifically, the crossover point $\tau_{s} \approx 316$, $258,232,283$, and 249 ps corresponding to the case of the experimental data shown as $\mathbf{\Delta}, \times, \square, \boldsymbol{\nabla}$ and $\mathbf{\square}$, respectively. We find that both the experimental data of Han and Kawakami ${ }^{37}$ and Avsar et al ${ }^{38}$ are larger than the crossover point whereas the one of Yang et al $\underline{\underline{66}}$ is smaller. This suggests the experimental data of Han and Kawakami ${ }^{37}$ and Avsar et al ${ }^{38}$ can be explained by the short-range scatterers. As for the experiment of Yang et $a l ., 66$ some other extrinsic effects have to be considered.

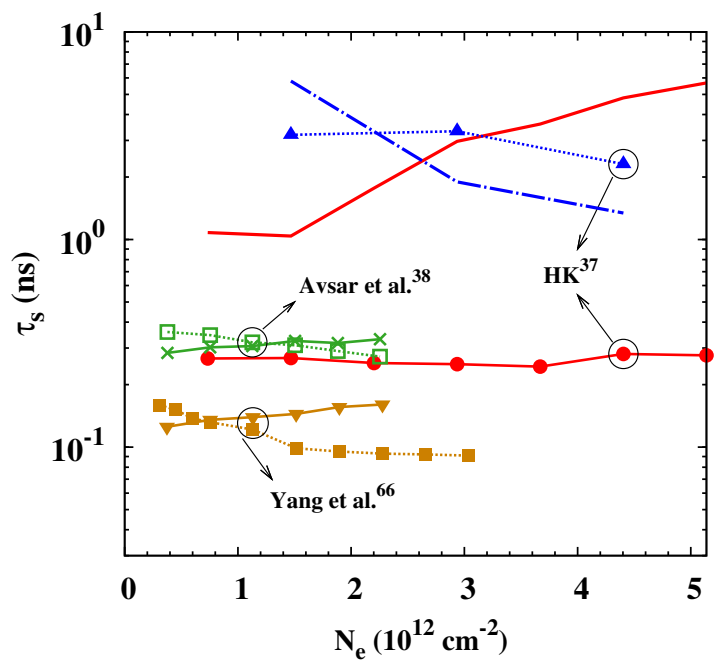

FIG. 6: (Color online) In-plane SRT $\tau_{s}$ as function of electron density. The dotted curves with symbols correspond to the experimental data of Han and Kawakami (HK) ${ }^{37}$ at $T=20 \mathrm{~K}$ and Avsar et al. ${ }^{38}$ and Yang et al. ${ }^{66}$ at $T=5 \mathrm{~K}$. The solid curves with symbols correspond to the experimental data at room temperature of Han and Kawakami, $\frac{37}{3}$ Avsar et al.$^{38}$ and Yang et al. ${ }^{66}$ Red solid (blue chain) curve is from our calculation corresponding to the case of room (low) temperature in the experiment of Han and Kawakami. ${ }^{37}$ It is noted that the blue chain curve is calculated with the short-range scatterers with the short-range impurity density $n_{d}=10^{10} \mathrm{~cm}^{-2}$ and potential strength $V_{0}=6 \times 10^{-17} \mathrm{meV} \mathrm{m}^{2}$.

Then we turn to compare the electron density dependence of the SRTs from the experiments and our calcu- lation. Among the experiments, Han and Kawakami ${ }^{37}$ measured the longest SRT at low temperature (up to several nanoseconds) shown as the dotted curve with $\boldsymbol{\Delta}$ in Fig. 6. This SRT is about one order of magnitude larger than the ones from other experimental cases. In addition, the SRTs from the experiments show different dependences on electron density. Specifically, at low temperature, both Avsar et al $\underline{38}$ and Yang et al s6 $^{66}$ show a decrease of the SRT with the increase of the electron density whereas a mild peak is observed by Han and Kawakami $: 37$ At room temperature, the SRTs from Avsar et $a l, \frac{38}{\underline{6}}$ and Yang et al $\underline{\underline{66}}$ increase with increasing electron density whereas Han and Kawakami37 reported an insensitive density dependence of the SRT.

We first show our comparison with the experimental data at low temperature. As shown in Fig. 5(a), our result is orders of magnitude larger than the experimental data of Han and Kawakami ${ }^{37}$ at low temperature without short-range impurities. However, with the inclusion of the short-range scatterers, our result shows good agreement with the experimental data. Here, with the short-range scatterers explicitly included, we also show our comparison with the experimental data of Han and Kawakami $\stackrel{37}{ }$ With a fixed short-range impurity density $n_{d}=10^{10} \mathrm{~cm}^{-2}$ and potential strength $V_{0}=6 \times 10^{-17} \mathrm{meV} \mathrm{m}^{2}$, the electron density dependence of the SRT from our calculation is shown as blue chain curve in Fig. 6. We find that our result marginally agrees with the experimental data shown as the dotted curve with $\boldsymbol{\Delta}$. Better agreement can be obtained with tunable short-range impurity density. In addition, we also find that the experimental data of Avsar et al $\underline{38}$ can be explained by the short-range scatterers. However, to account for the experimental data of Yang et al. $\frac{66}{,}$ one has to include other extrinsic effects. This is because the SRT of Yang et al $\underline{66}$ is smaller than the crossover point in the short-range impurity density dependence of the SRT (230-320 ps), which is taken as a criterion for the possible contribution of the short-range scattering as mentioned previously.

We then address our comparison with the experimental data at room temperature. As shown in Fig. 5(a), our result is larger than the experimental data of Han and Kawakami ${ }^{37}$ with an observable difference at room temperature without short-range impurities. With the short-range scatterers included, the SRT from our calculation becomes even larger than the experimental data as mentioned previously, indicating that other extrinsic effects have to be taken into account. Here, we show our comparison with the experiment of Han and Kawakami ${ }^{37}$ without extrinsic effects. The experimental data and our result are shown as the solid curve with $\bullet$ and the red solid curve in Fig. 6. respectively. We find that the SRT in our calculation is about three times as large as the experimental one in low electron density regime and one order of magnitude larger than the experimental one in high density regime. 


\section{SUMMARY}

In summary, we have investigated the electron spin relaxation due to the DP mechanism in BLG with only the lowest conduction band being relevant. The SOC of the lowest conduction band is constructed from the symmetry group analysis with the parameters obtained by fitting to the numerical calculation according to the latest report by Konschuh et al. .55 from first principles with both the intrinsic and extrinsic SOC terms in the pseudospin space included. We find that the magnitudes of both the out-of- and in-plane components of the SOC decrease with increasing momentum at large momentum, indicating a suppression of the inhomogeneous broadening with the increase of the momentum. This is different from the case in both semiconductors and single-layer graphene. Additionally, the leading term of the out-of-plane component of the SOC serves as a Zeeman-like term with opposite effective magnetic fields in the two valleys, which is similar to the case in rippled single-layer graphene. This Zeeman-like term, together with the intervalley electron-phonon and/or possible intervalley short-range scatterings, opens an intervalley spin relaxation channel, which has not been reported in the literature in BLG. The intervalley electronphonon scattering is derived by using the tight-binding model. In addition to the intervalley electron-phonon and short-range scatterings, we also include the longrange electron-impurity, electron-electron Coulomb and intravalley electron-phonon scatterings to calculate the SRT. We find that the in-plane SRT is strongly suppressed by the intervalley electron-phonon scattering at high temperature. In contrast to the intervalley electronphonon scattering, the intervalley short-range scattering is insensitive to the temperature and plays an important role in the in-plane spin relaxation especially at low temperature.

In the absence of short-range scatterers, a marked nonmonotonic temperature dependence of the in-plane SRT is predicted with a minimum SRT down to several hundred picoseconds. This nonmonotonic behavior originates from the crossover between the weak and strong intervalley electron-phonon scattering. Moreover, we predict a peak in the electron density dependence of the in-plane SRT at low temperature, which is very different from the one in semiconductors. At high temperature, the in-plane SRT increases monotonically with increasing density. We also find that the in-plane SRT decreases rapidly with the increase of the initial spin polarization at low temperature. This is very different from the previous studies in both semiconductors and single-layer graphene where the SRT increases significantly with increasing initial spin polarization. The physics is understood that the spin relaxation time is determined by the intervalley electron-phonon scattering, which transfers electrons between the two valleys. The transferred electrons experience opposite effective magnetic fields in the two valleys, which are not affected by the Coulomb HF term.
In addition, a strong anisotropy between the out-of- and in-plane spin relaxations is also addressed at high temperature where the out-of-plane SRT is about two orders of magnitude larger than the in-plane one. As for low temperature, the out-of- and in-plane spin relaxation times are comparable.

We also show our comparison with the existing experiments of Han and Kawakami, 37 Avsar et al. ${ }^{38}$ and Yang et al..$^{66}$ We find that without intervalley scattering, the SRT is orders of magnitude larger than the experimental data in the whole temperature regime. With the intervalley electron-phonon scattering explicitly included, the SRT from our calculation becomes comparable to the experimental data at high temperature but still orders of magnitude larger than the experimental data at low temperature. In addition, a crossover in the temperature dependence of the in-plane SRT is shown, which also results from the crossover between the weak and strong intervalley electron-phonon scattering. With the inclusion of the short-range scatterers, our result agrees fairly well with the experimental data in the temperature regime lower than the crossover. As for the temperature higher than the crossover, other extrinsic effects have to be included. Moreover, a crossover point is also shown in the shortrange impurity density dependence of the in-plane SRT, which gives a minimum for determining the possible contribution of the short-range scattering. The experimental SRT larger than the minimum can be explained by the possible short-range scatterers whereas other extrinsic effects have to be considered for experimental data smaller than the minimum.

\section{Acknowledgments}

This work was supported by the National Basic Research Program of China under Grant No. 2012CB922002 and the Strategic Priority Research Program of the Chinese Academy of Sciences under Grant No. XDB01000000. One of the authors (L.W.) would like to thank B. Y. Sun for double checking the derivation of the electron-phonon scattering matrix elements.

\section{Appendix A: SOC OF THE LOWEST CONDUCTION BAND}

The SOC term of the lowest conduction band near the Dirac points can be described by a $2 \times 2$ matrix

$$
H_{\mathrm{eff} \mu}^{\mathrm{SO}}=\left(\begin{array}{cc}
H_{\mu 11}^{\mathrm{SO}} & H_{\mu 12}^{\mathrm{SO}} \\
H_{\mu 12}^{\mathrm{SO}} & H_{\mu 22}^{\mathrm{SO}}
\end{array}\right)
$$

in the basis $\psi_{\mathbf{k} s}^{\mu}=\psi_{\mathbf{k}}^{\mu} \otimes|s\rangle$ where $s=\{\uparrow, \downarrow\}$ and $\psi_{\mathbf{k}}^{\mu}=$ $\sum_{i} c_{i}^{\mu}(\mathbf{k}) \Psi_{i}^{\mu}(\mathbf{k})\left(i=\mathrm{A}_{1}, \mathrm{~B}_{1}, \mathrm{~A}_{2}, \mathrm{~B}_{2}\right)$. The coefficients $c_{i}^{\mu}(\mathbf{k})$ can be obtained by exactly diagonalizing the $4 \times 4$ 

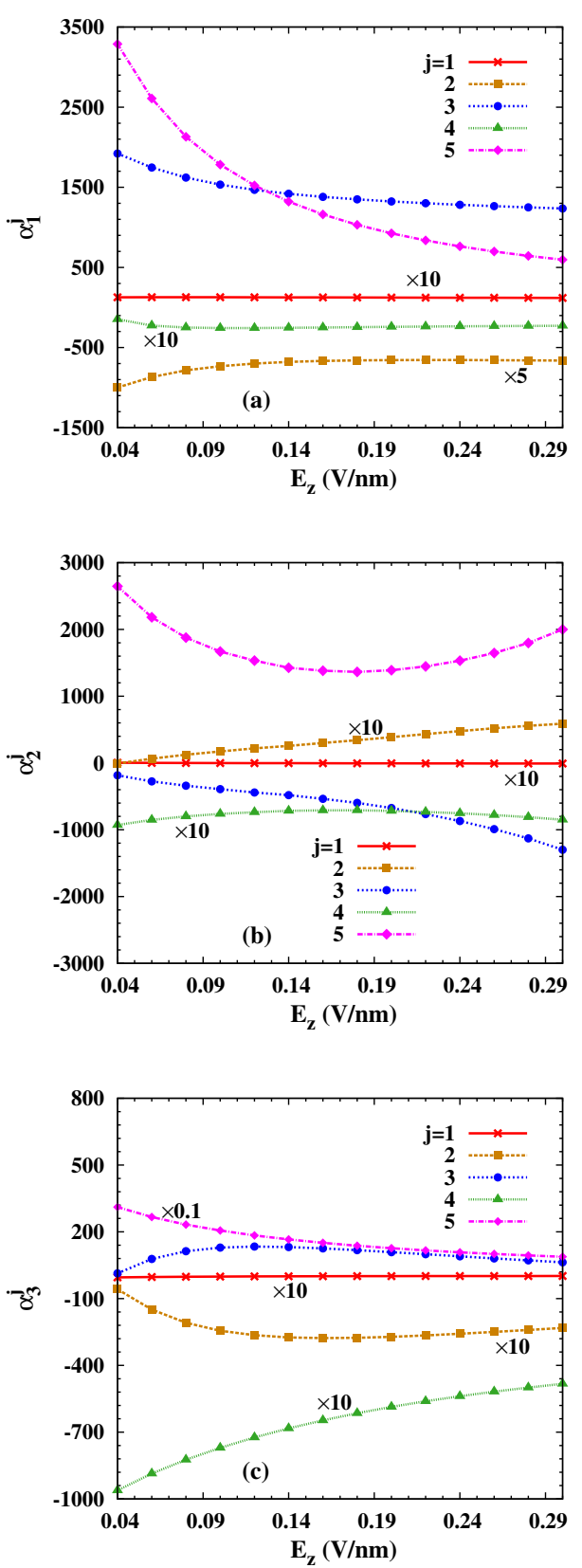

FIG. 7: (Color online) (a) $\alpha_{1}^{j}$, (b) $\alpha_{2}^{j}$ and (c) $\alpha_{3}^{j}$ as function of the out-of-plane electric field $E_{z}$ with $j=1-5$. It is noted that $\alpha_{i}^{1}, \alpha_{i}^{2}, \alpha_{i}^{3}, \alpha_{i}^{4}$ and $\alpha_{i}^{5}(i=1-3)$ are dimensionless.

effective Hamiltonian given in Eq. (5). Here,

$$
\begin{aligned}
& H_{\mu 11}^{\mathrm{SO}}=\sum_{i, j} c_{i}^{\mu *}(\mathbf{k}) c_{j}^{\mu}(\mathbf{k})\left\langle\Psi_{i, \uparrow}^{\mu}\left|H_{\mathrm{SO}}\right| \Psi_{j, \uparrow}^{\mu}\right\rangle, \\
& H_{\mu 22}^{\mathrm{SO}}=\sum_{i, j} c_{i}^{\mu *}(\mathbf{k}) c_{j}^{\mu}(\mathbf{k})\left\langle\Psi_{i, \downarrow}^{\mu}\left|H_{\mathrm{SO}}\right| \Psi_{j, \downarrow}^{\mu}\right\rangle, \\
& H_{\mu 12}^{\mathrm{SO}}=\sum_{i, j} c_{i}^{\mu *}(\mathbf{k}) c_{j}^{\mu}(\mathbf{k})\left\langle\Psi_{i, \uparrow}^{\mu}\left|H_{\mathrm{SO}}\right| \Psi_{j, \downarrow}^{\mu}\right\rangle,
\end{aligned}
$$

with $\left\langle\Psi_{i, s_{1}}^{\mu}\left|H_{\mathrm{SO}}\right| \Psi_{j, s_{2}}^{\mu}\right\rangle\left(s_{1,2}=\{\uparrow, \downarrow\}\right)$ being the spinorbit matrix elements near the Dirac points in the pseudospin space given by Konschuh et al. $\frac{55}{5}$ Specifically,

$$
\begin{aligned}
H_{\mu 11}^{\mathrm{SO}} & =\mu \lambda_{\mathrm{I} 1}\left(\left|c_{3}^{\mu}\right|^{2}-\left|c_{2}^{\mu}\right|^{2}\right)+\mu \lambda_{\mathrm{I} 2}\left(\left|c_{1}^{\mu}\right|^{2}-\left|c_{4}^{\mu}\right|^{2}\right) \\
H_{\mu 22}^{\mathrm{SO}} & =-H_{\mu 11}^{\mathrm{SO}} \\
H_{\mu 12}^{\mathrm{SO}} & =\frac{\mu+1}{2}\left[c_{1}^{\mu *} c_{3}^{\mu}\left(i \lambda_{4}\right)+c_{2}^{\mu *} c_{1}^{\mu}\left(-i \lambda_{0}\right)+c_{2}^{\mu *} c_{4}^{\mu}\left(-i \lambda_{4}^{\prime}\right)\right. \\
& \left.+c_{3}^{\mu *} c_{2}^{\mu}\left(-i \lambda_{3}\right)+c_{4}^{\mu *} c_{3}^{\mu}\left(i \lambda_{0}^{\prime}\right)\right]+\frac{\mu-1}{2}\left[c_{3}^{\mu *} c_{1}^{\mu}\left(i \lambda_{4}\right)\right. \\
& +c_{1}^{\mu *} c_{2}^{\mu}\left(-i \lambda_{0}\right)+c_{4}^{\mu *} c_{2}^{\mu}\left(-i \lambda_{4}^{\prime}\right)+c_{2}^{\mu *} c_{3}^{\mu}\left(-i \lambda_{3}\right) \\
& \left.+c_{3}^{\mu *} c_{4}^{\mu}\left(i \lambda_{0}^{\prime}\right)\right] .
\end{aligned}
$$

$\lambda_{\mathrm{I} 1}$ and $\lambda_{\mathrm{I} 2}$ are the strengths of single-layer-like intrinsic SOC; $\lambda_{0}$ and $\lambda_{0}^{\prime}$ represent single-layer-like extrinsic spinorbit strengths; $\lambda_{3}, \lambda_{4}$ and $\lambda_{4}^{\prime}$ stand for interlayer spinorbit parameters $\frac{55}{5}$ Then the effective magnetic field of the SOC $\boldsymbol{\Omega}^{\mu}(\mathbf{k})$ in Eq. (6) is given by $\Omega_{x}^{\mu}(\mathbf{k})=2 \operatorname{Re} H_{\mu 12}^{\mathrm{SO}}$, $\Omega_{y}^{\mu}(\mathbf{k})=-2 \operatorname{Im} H_{\mu 12}^{\mathrm{SO}}$ and $\Omega_{z}^{\mu}(\mathbf{k})=2 H_{\mu 11}^{\mathrm{SO}}$. It is noted that the contribution of other energy bands to the SOC of the lowest conduction band is marginal in our calculation.

As reported by Konschuh et al.,, 55 the small group of the Dirac points is $C_{3}$ in the presence of an out-of-plane electric field. Following from the symmetry group $C_{3} \stackrel{78}{\underline{78}}$ we derive an analytical form of the SOC near the Dirac points [see Eqs. (113)]. In these equations, the coefficients $\alpha_{i}(k)(i=1-3)$ and $\beta_{i}(k)(i=1-2)$ are obtained by fitting to the numerical calculation from Eqs. A5 and (A7) using the Padé approximation ${ }^{94}$ Specifically, the coefficients $\alpha_{i}(k)(i=1-3)$ in Eqs. (1-2) read

$$
\alpha_{i}(k)=\lambda_{\mathrm{I} 1} a k \frac{\alpha_{i}^{1}+\alpha_{i}^{2} a k+\alpha_{i}^{3} a^{2} k^{2}}{1+\alpha_{i}^{4} a k+\alpha_{i}^{5} a^{2} k^{2}} .
$$

The coefficients $\beta_{i}(k)(i=1-2)$ in Eq. (3) are given by

$$
\begin{aligned}
& \beta_{1}(k)=\beta_{1}^{0}+\lambda_{\mathrm{I} 1} a k \frac{\beta_{1}^{1}+\beta_{1}^{2} a k}{1+\beta_{1}^{3} a k+\beta_{1}^{4} a^{2} k^{2}}, \\
& \beta_{2}(k)=\lambda_{\mathrm{I} 1} a k \frac{\beta_{2}^{1}+\beta_{2}^{2} a k}{1+\beta_{2}^{3} a k+\beta_{2}^{4} a^{2} k^{2}} .
\end{aligned}
$$

Here, the coefficient $\beta_{1}^{0}=-24 \mu \mathrm{eV}$, which is independent of the applied electric field. The electric field dependences of $\alpha_{i}^{j}(i=1-3, j=1-5)$ and $\beta_{i}^{j}(i=1-2$, $j=1-4)$ are plotted in Figs. 7 and 8 respectively. It is noted that the Padé approximation ${ }^{94}$ can give a precise description of $\alpha_{i}(k)(i=1-3)$ and $\beta_{i}(k)(i=1-2)$ at large momenta near the Dirac points, which can be populated and play an important role in spin relaxation according to the experimental conditions (heavily doped) $37,38,66,68$ With only a simple linear $k$-order approximation, $\alpha_{i}(k)$ and $\beta_{i}(k)$ agree with the numerical results only at very small momentum near the Dirac points.

We also show in Fig. 9 the spin splitting of the lowest conduction band calculated with analytical form of the SOC (blue dashed curve) and with the explicit numerical one (red solid curve). We find that the analytical 

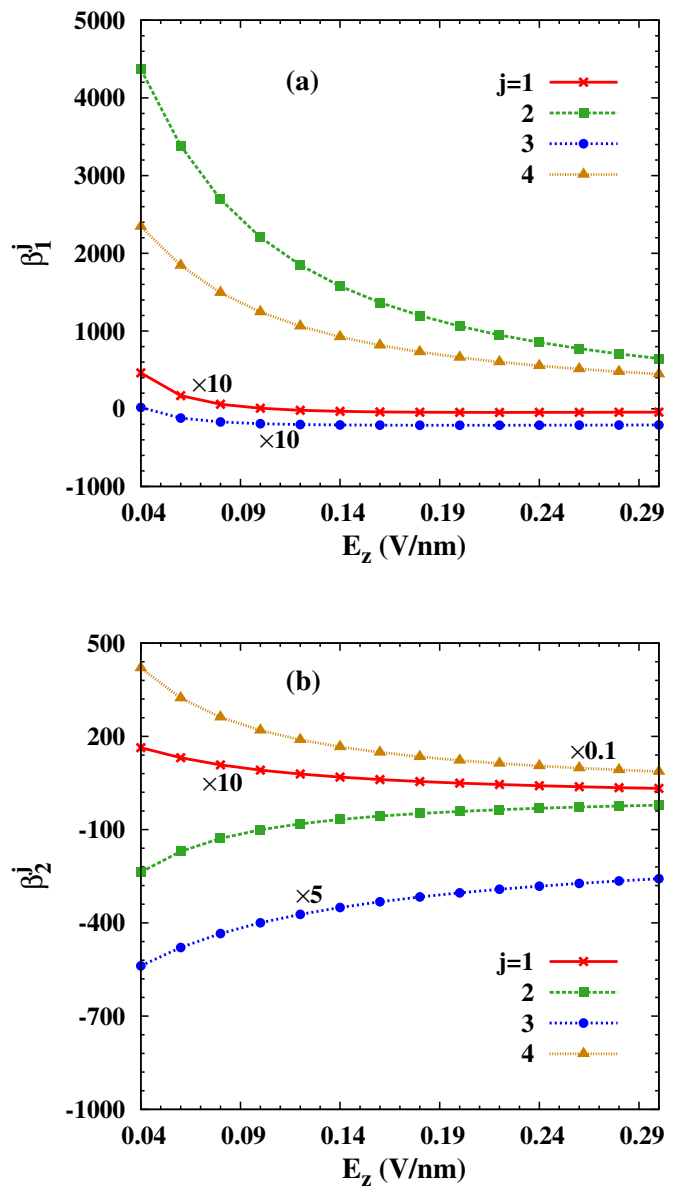

FIG. 8: (Color online) (a) $\beta_{1}^{j}$ and (b) $\beta_{2}^{j}$ as function of the out-of-plane electric field $E_{z}$ with $j=1-4$. It is noted that $\beta_{i}^{1}, \beta_{i}^{2}, \beta_{i}^{3}$ and $\beta_{i}^{4}(i=1,2)$ are dimensionless.

result agrees fairly well with the numerical one at large momentum. In addition, the spin splitting decreases with increasing momentum at large momentum, which is very different from the case in both semiconductors ${ }^{70}$ and single-layer graphene $\underline{36}$

\section{Appendix B: SCATTERING MATRIX ELEMENTS}

The electron-electron Coulomb scattering matrix element is given by $\left|V_{\mathbf{k}, \mathbf{k}-\mathbf{q}}^{\mu}\right|^{2}$. The screened Coulomb potential $V_{\mathbf{k}, \mathbf{k}-\mathbf{q}}^{\mu}=V_{\mathbf{q}}^{(0)} / \varepsilon\left(\mathbf{q}, \epsilon_{\mu \mathbf{k}}-\epsilon_{\mu \mathbf{k}-\mathbf{q}}\right)$ with $\varepsilon\left(\mathbf{q}, \epsilon_{\mu \mathbf{k}}-\right.$ $\left.\epsilon_{\mu \mathbf{k}-\mathbf{q}}\right)=1-V_{\mathbf{q}}^{(0)} \Pi\left(\mathbf{q}, \epsilon_{\mu \mathbf{k}}-\epsilon_{\mu \mathbf{k}-\mathbf{q}}\right)$ being the screening under the random phase approximation. ${ }^{95} V_{\mathbf{q}}^{(0)}=$ $2 \pi v_{\mathrm{F}} r_{s} / q$ is the two-dimensional bare Coulomb potential with $v_{\mathrm{F}}$ and $r_{s}$ being the Fermi velocity in singlelayer graphene ${ }^{87}$ and the dimensionless Wigner-Seitz

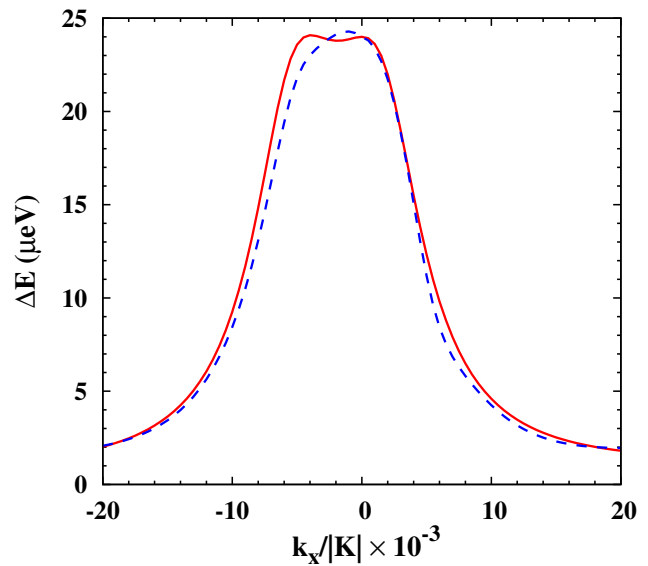

FIG. 9: (Color online) Spin splitting of the lowest conduction band $\Delta E$ near the $\mathrm{K}$ point as function of the momentum $k_{x}$ under a typical electric field $E_{z}=0.14 \mathrm{~V} / \mathrm{nm}$. Solid (dashed) curve represents the numerical (analytical) result. $|\mathrm{K}|=4 \pi /(3 a)$.

radius,$\stackrel{96-98}{-2 e s p e c t i v e l y . ~} \Pi(\mathbf{q}, \omega)$ is given by $36,99-101$

$\Pi(\mathbf{q}, \omega)=\sum_{\mu \mathbf{k} \nu \nu^{\prime} s}\left|T_{\mathbf{k k}+\mathbf{q}}^{\mu \nu \nu^{\prime}}\right|^{2} \frac{f_{\mathbf{k} s}^{\mu \nu}-f_{\mathbf{k}+\mathbf{q} s}^{\mu \nu^{\prime}}}{\epsilon_{\mu \nu \mathbf{k}}-\epsilon_{\mu \nu^{\prime} \mathbf{k}+\mathbf{q}}+\omega+i 0^{+}}$,

where $T_{\mathbf{k k}+\mathbf{q}}^{\mu \nu \nu^{\prime}}=\psi_{\mathbf{k}}^{\mu \nu \dagger} \psi_{\mathbf{k}+\mathbf{q}}^{\mu \nu^{\prime}}$. The long-range electronimpurity scattering matrix element $\left|U_{\mathbf{k}, \mathbf{k}-\mathbf{q}}^{\mu}\right|^{2}=$ $Z_{i}^{2}\left|V_{\mathbf{k}, \mathbf{k}-\mathbf{q}}^{\mu}\right|^{2} e^{-2 q d}$, in which $Z_{i}$ and $d$ stand for the impurity charge number and effective distance of the impurity layer to the BLG sheet $\underline{44,96-98,102}$ It is noted that when we calculate the electron-electron Coulomb and long-range electron-impurity scatterings, we take the distance between the graphene layers to be zero approximately $\frac{103}{1}$ It is further noted that the Coulomb potential in the HF term is in the static screening limit, i.e., $V_{\mathbf{k}, \mathbf{k}-\mathbf{q}}^{\mu}=V_{\mathbf{q}}^{(0)} / \varepsilon(\mathbf{q}, 0)$.

Then we turn to the electron-phonon scattering matrix elements. For the intravalley electron-AC-phonon scattering, the scattering matrix element $\left|M_{\mu \mathbf{k}, \mu^{\prime} \mathbf{k}^{\prime}}^{\mathrm{AC}}\right|^{2}=$ $D_{\mathrm{AC}}^{2} \hbar q /\left(2 \rho v_{\mathrm{ph}}\right) I_{\mathbf{k k}^{\prime}}^{\mu} \delta_{\mu \mu^{\prime}}$ where $q=\left|\mathbf{k}-\mathbf{k}^{\prime}\right| ; D_{\mathrm{AC}}$ and $v_{\text {ph }}$ represent the deformation potential ${ }^{64}$ and acoustic phonon velocity $\underset{104,105}{i}$ respectively.

For the intravalley electron-RI-phonon scattering, $\left|M_{\mu \mathbf{k}, \mu^{\prime} \mathbf{k}^{\prime}}^{\mathrm{RI}}\right|^{2}=g \frac{\sqrt{3} \hbar^{2} v_{\mathrm{F}}^{2}}{a} \frac{e^{-2 q d}}{q+q_{s}} I_{\mathbf{k k}^{\prime}}^{\mu} \delta_{\mu \mu^{\prime}}$ with $q_{s}=4 r_{s} k_{\mathrm{F}}$ being the Thomas-Fermi screening length. $\underline{\underline{99}}$ For $\mathrm{SiO}_{2}$ substrate, the energy spectra of the two remote phonon modes are denoted as $\omega_{1}^{\mathrm{RI}}$ and $\omega_{2}^{\mathrm{RI}}$, respectively; $g_{1}$ and $g_{2}$ are the corresponding dimensionless coupling parameters $\frac{102}{1}$

The matrix elements for the intravalley electron-OPphonon scattering $\left(\left|M_{\mu \mathbf{k}, \mu^{\prime} \mathbf{k}^{\prime}}^{\mathrm{OP}}\right|^{2}\right)$ are derived using the tight-binding model according to the arXiv version of Ref. 63. The intravalley electron-OP-phonon scattering 
includes both the electron in-plane $\left(\left|M_{\mu \mathbf{k}, \mu^{\prime} \mathbf{k}^{\prime}}^{\mathrm{LT}}\right|^{2}\right)$ and outof-plane $\left(\left|M_{\mu \mathbf{k}, \mu^{\prime} \mathbf{k}^{\prime}}^{\mathrm{ZO}}\right|^{2}\right)$ OP phonon scatterings. The electron in-plane OP phonon scattering matrix element is given by

$$
\begin{aligned}
& \left|M_{\mu \mathbf{k}, \mu^{\prime} \mathbf{k}^{\prime}}^{\mathrm{LT}}\right|^{2}=\frac{9 \hbar^{2}}{2 \rho \Omega_{\mathrm{LT}}} \delta_{\mu \mu^{\prime}}\left\{\mid \psi_{\mathbf{k}}^{\mu \dagger}\left[\gamma_{0}^{\prime}\left(\sin \theta_{\mathbf{q}} \sigma_{\mathrm{D}}^{23}+\cos \theta_{\mathbf{q}} \sigma_{\mathrm{D}}^{13}\right)\right.\right. \\
& \quad-a \gamma_{3}^{\prime}\left(\sin \theta_{\mathbf{q}} \gamma_{\mathrm{D}}^{1} \gamma_{\mathrm{D}}^{5}-i \sin \theta_{\mathbf{q}} \gamma_{\mathrm{D}}^{2}+i \cos \theta_{\mathbf{q}} \gamma_{\mathrm{D}}^{1}+\cos \theta_{\mathbf{q}}\right. \\
& \left.\left.\quad \times \gamma_{\mathrm{D}}^{2} \gamma_{\mathrm{D}}^{5}\right) /\left(2 \sqrt{3} l_{3}\right)\right]\left.\psi_{\mathbf{k}^{\prime}}^{\mu}\right|^{2}+\mid \psi_{\mathbf{k}}^{\mu \dagger}\left[i \gamma _ { 0 } ^ { \prime } \left(\sin \theta_{\mathbf{q}} \sigma_{\mathrm{D}}^{01}-\cos \theta_{\mathbf{q}}\right.\right. \\
& \left.\left.\quad \times \sigma_{\mathrm{D}}^{02}\right)+a \gamma_{4}^{\prime}\left(i \cos \theta_{\mathbf{q}} \gamma_{\mathrm{D}}^{3}-\sin \theta_{\mathbf{q}} \gamma_{\mathrm{D}}^{3} \gamma_{\mathrm{D}}^{5}\right) /\left(\sqrt{3} l_{4}\right)\right]\left.\psi_{\mathbf{k}^{\prime}}^{\mu}\right|^{2} \\
& \quad+\mid \psi_{\mathbf{k}}^{\mu \dagger}\left[\gamma_{0}^{\prime}\left(\cos \theta_{\mathbf{q}} \sigma_{\mathrm{D}}^{23}-\sin \theta_{\mathbf{q}} \sigma_{\mathrm{D}}^{13}\right)-a \gamma_{3}^{\prime}\left(\cos \theta_{\mathbf{q}} \gamma_{\mathrm{D}}^{1} \gamma_{\mathrm{D}}^{5}\right.\right. \\
& \left.\left.\quad-i \cos \theta_{\mathbf{q}} \gamma_{\mathrm{D}}^{2}-i \sin \theta_{\mathbf{q}} \gamma_{\mathrm{D}}^{1}-\sin \theta_{\mathbf{q}} \gamma_{\mathrm{D}}^{2} \gamma_{\mathrm{D}}^{5}\right) /\left(2 \sqrt{3} l_{3}\right)\right]\left.\psi_{\mathbf{k}^{\prime}}^{\mu}\right|^{2} \\
& \quad+\mid \psi_{\mathbf{k}}^{\mu \dagger}\left[i \gamma_{0}^{\prime}\left(\cos \theta_{\mathbf{q}} \sigma_{\mathrm{D}}^{01}+\sin \theta_{\mathbf{q}} \sigma_{\mathrm{D}}^{02}\right)-a \gamma_{4}^{\prime} \gamma_{\mathrm{D}}^{3}\left(i \sin \theta_{\mathbf{q}}\right.\right. \\
& \left.\left.\left.\quad+\cos \theta_{\mathbf{q}} \gamma_{\mathrm{D}}^{5}\right) /\left(\sqrt{3} l_{4}\right)\right]\left.\psi_{\mathbf{k}^{\prime}}^{\mu}\right|^{2}\right\}
\end{aligned}
$$

where $\Omega_{\mathrm{LT}}$ is the energy of the in-plane OP phonon modes; $83,84 l_{3}$ is the bond length corresponding to the interlayer hopping $\gamma_{3}$; and $\sigma_{\mathrm{D}}^{02}$ and $\sigma_{\mathrm{D}}^{13}$ are $4 \times 4$ Dirac matrices given in Appendix [C ${ }^{86}$ Here, $\theta_{\mathbf{q}}$ is the polar angle of the momentum q. The electron out-of-plane OP phonon scattering matrix element reads

$$
\left|M_{\mu \mathbf{k}, \mu^{\prime} \mathbf{k}^{\prime}}^{\mathrm{ZO}}\right|^{2}=\frac{\hbar^{2}{\gamma_{1}^{\prime}}^{2}}{2 \rho \Omega_{\mathrm{ZO}}} \delta_{\mu \mu^{\prime}}\left|\psi_{\mathbf{k}}^{\mu \dagger}\left(\gamma_{\mathrm{D}}^{1} \gamma_{\mathrm{D}}^{5}+i \gamma_{\mathrm{D}}^{2}\right) \psi_{\mathbf{k}^{\prime}}^{\mu}\right|^{2}
$$

with $\Omega_{\mathrm{ZO}}$ being the energy of the out-of-plane OP phonon mode 64

\section{Appendix C: RELEVANT DIRAC MATRICES}

The relevant Dirac matrices ${ }^{86}$ used in the electronphonon scattering are

$$
\begin{gathered}
\gamma_{\mathrm{D}}^{0}=\left(\begin{array}{ll}
0 & I \\
I & 0
\end{array}\right), \quad \gamma_{\mathrm{D}}^{5}=\left(\begin{array}{cc}
-I & 0 \\
0 & I
\end{array}\right) \\
\sigma_{\mathrm{D}}^{01}=-i\left(\begin{array}{cc}
\sigma_{x} & 0 \\
0 & -\sigma_{x}
\end{array}\right), \sigma_{\mathrm{D}}^{13}=-\left(\begin{array}{cc}
\sigma_{y} & 0 \\
0 & \sigma_{y}
\end{array}\right), \\
\sigma_{\mathrm{D}}^{02}=-i\left(\begin{array}{cc}
\sigma_{y} & 0 \\
0 & -\sigma_{y}
\end{array}\right), \sigma_{\mathrm{D}}^{23}=\left(\begin{array}{cc}
\sigma_{x} & 0 \\
0 & \sigma_{x}
\end{array}\right)
\end{gathered}
$$

$$
\gamma_{\mathrm{D}}^{i}=\left(\begin{array}{cc}
0 & \sigma_{i} \\
-\sigma_{i} & 0
\end{array}\right) \quad(i=1-3)
$$

with $I$ being $2 \times 2$ unit matrix.
* Author to whom correspondence should be addressed; Electronic address: mwwu@ustc.edu.cn.

1 K. S. Novoselov, A. K. Geim, S. V. Morozov, D. Jiang, Y. Zhang, S. V. Dubonos, I. V. Grigorieva, and A. A. Firsov, Science 306, 666 (2004).

${ }^{2}$ A. K. Geim and K. S. Novoselov, Nature Mater. 6, 183 (2007).

3 N. Tombros, C. Jozsa, M. Popinciuc, H. T. Jonkman, and B. J. van Wees, Nature (London) 448, 571 (2007).

${ }^{4}$ F. Wang, Y. Zhang, C. Tian, C. Girit, A. Zettl, M. Crommie, and Y. Ron Shen, Science 320, 206 (2008).

${ }^{5}$ C. W. J. Beenakker, Rev. Mod. Phys. 80, 1337 (2008).

6 A. H. Castro Neto, F. Guinea, N. M. R. Peres, K. S. Novoselov, and A. K. Geim, Rev. Mod. Phys. 81, 109 (2009).

7 N. M. R. Peres, Rev. Mod. Phys. 82, 2673 (2010).

8 D. S. L. Abergel, V. Apalkov, J. Berashevich, K. Ziegler, and T. Chakraborty, Adv. Phys. 59, 261 (2010).

9 S. Das Sarma, S. Adam, E. H. Hwang, and E. Rossi, Rev. Mod. Phys. 83, 407 (2011).

10 M. Acik and Y. J. Chabal, Jpn. J. Appl. Phys. 50, 070101 (2011).

11 A. K. Geim, Rev. Mod. Phys. 83, 851 (2011).

12 M. O. Goerbig, Rev. Mod. Phys. 83, 1193 (2011).

13 V. N. Kotov, B. Uchoa, V. M. Pereira, F. Guinea, and A. H. Castro Neto, Rev. Mod. Phys. 84, 1067 (2012).

14 D. R. Cooper, B. D'Anjou, N. Ghattamaneni, B. Harack, M. Hilke, A. Horth, N. Majlis, M. Massicotte, L. Vands- burger, E. Whiteway, and V. Yu, ISRN Condensed Matter Physics 2012, 501686 (2012).

15 C. L. Kane and E. J. Mele, Phys. Rev. Lett. 95, 226801 (2005).

16 D. Huertas-Hernando, F. Guinea, and A. Brataas, Phys. Rev. B 74, 155426 (2006).

17 H. Min, J. E. Hill, N. A. Sinitsyn, B. R. Sahu, L. Kleinman, and A. H. MacDonald, Phys. Rev. B 74, 165310 (2006).

18 Y. Yao, F. Ye, X.-L. Qi, S.-C. Zhang, and Z. Fang, Phys. Rev. B 75, 041401(R) (2007).

19 J. C. Boettger and S. B. Trickey, Phys. Rev. B 75, 121402(R) (2007).

20 B. Trauzettel, D. V. Bulaev, D. Loss, and G. Burkard, Nature Phys. 3, 192 (2007).

21 J. Fischer, B. Trauzettel, and D. Loss, Phys. Rev. B 80, 155401 (2009).

22 M. Gmitra, S. Konschuh, C. Ertler, C. Ambrosch-Draxl, and J. Fabian, Phys. Rev. B 80, 235431 (2009).

23 D. Huertas-Hernando, F. Guinea, and A. Brataas, Phys. Rev. Lett. 103, 146801 (2009).

24 S. Abdelouahed, A. Ernst, J. Henk, I. V. Maznichenko, and I. Mertig, Phys. Rev. B 82, 125424 (2010).

25 S. Konschuh, M. Gmitra, and J. Fabian, Phys. Rev. B 82, 245412 (2010).

26 S. Cho, Y.-F. Chen, and M. S. Fuhrer, Appl. Phys. Lett. 91, 123105 (2007).

27 C. Józsa, M. Popinciuc, N. Tombros, H. T. Jonkman, and 
B. J. van Wees, Phys. Rev. Lett. 100, 236603 (2008).

28 N. Tombros, S. Tanabe, A. Veligura, C. Józsa, M. Popinciuc, H. T. Jonkman, and B. J. van Wees, Phys. Rev. Lett. 101, 046601 (2008).

29 C. Józsa, T. Maassen, M. Popinciuc, P. J. Zomer, A. Veligura, H. T. Jonkman, and B. J. van Wees, Phys. Rev. B 80, 241403(R) (2009).

30 M. Popinciuc, C. Józsa, P. J. Zomer, N. Tombros, A. Veligura, H. T. Jonkman, and B. J. van Wees, Phys. Rev. B 80, 214427 (2009).

31 W. Han, K. Pi, W. Bao, K. M. McCreary, Y. Li, W. H. Wang, C. N. Lau, and R. K. Kawakami, Appl. Phys. Lett. 94, 222109 (2009).

32 W. Han, W. H. Wang, K. Pi, K. M. McCreary, W. Bao, Y. Li, F. Miao, C. N. Lau, and R. K. Kawakami, Phys. Rev. Lett. 102, 137205 (2009).

33 M. Shiraishi, M. Ohishi, R. Nouchi, N. Mitoma, T. Nozaki, T. Shinjo, and Y. Suzuki, Adv. Funct. Mater. 19, 3711 (2009).

${ }^{34}$ K. Pi, W. Han, K. M. McCreary, A. G. Swartz, Y. Li, and R. K. Kawakami, Phys. Rev. Lett. 104, 187201 (2010).

35 W. Han, K. Pi, K. M. McCreary, Y. Li, J. J. I. Wong, A. G. Swartz, and R. K. Kawakami, Phys. Rev. Lett. 105, 167202 (2010).

${ }^{36}$ Y. Zhou and M. W. Wu, Phys. Rev. B 82, 085304 (2010).

37 W. Han and R. K. Kawakami, Phys. Rev. Lett. 107, 047207 (2011).

38 A. Avsar, T.-Y. Yang, S. Bae, J. Balakrishnan, F. Volmer, M. Jaiswal, Z. Yi, S. R. Ali, G. Güntherodt, B. H. Hong, B. Beschoten, and B. Özyilmaz, Nano Lett. 11, 2363 (2011).

39 W. Han, K. M. McCreary, K. Pi, W. H. Wang, Y. Li, H. Wen, J. R. Chen, and R. K. Kawakami, J. Magn. Magn. Mater. 324, 369 (2012).

40 T. Maassen, I. J. Vera-Marun, M. H. D. Guimarães, and B. J. van Wees, Phys. Rev. B 86, 235408 (2012).

41 M. Wojtaszek, I. J. Vera-Marun, T. Maassen, and B. J. van Wees, Phys. Rev. B 87, 081402(R) (2013).

42 A. Varykhalov, J. S. Barriga, A. M. Shikin, C. Biswas, E. Vescovo, A. Rybkin, D. Marchenko, and O. Rader, Phys. Rev. Lett. 101, 157601 (2008).

43 A. H. Castro Neto and F. Guinea, Phys. Rev. Lett. 103 026804 (2009)

44 C. Ertler, S. Konschuh, M. Gmitra, and J. Fabian, Phys. Rev. B 80, 041405(R) (2009).

45 V. K. Dugaev, E. Ya. Sherman, and J. Barnaś, Phys. Rev. B 83, 085306 (2011).

46 P. Zhang and M. W. Wu, Phys. Rev. B 84, 045304 (2011).

47 P. Zhang and M. W. Wu, New J. Phys. 14, 033015 (2012).

48 S. V. Morozov, K. S. Novoselov, M. I. Katsnelson, F. Schedin, L. A. Ponomarenko, D. Jiang, and A. K. Geim, Phys. Rev. Lett. 97, 016801 (2006).

49 J. S. Jeong, J. Shin, and H. W. Lee, Phys. Rev. B 84, 195457 (2011).

${ }^{50}$ P. Zhang, Y. Zhou, and M. W. Wu, J. Appl. Phys. 112, 073709 (2012).

51 Y. S. Dedkov, M. Fonin, U. Rüdiger, and C. Laubschat, Phys. Rev. Lett. 100, 107602 (2008).

52 S. Ryu, L. Liu, S. Berciaud, Y.-J. Yu, H. Liu, P, Kim, G. W. Flynn, and L. E. Brus, Nano Lett. 10, 4944 (2010).

53 P. Gallo, A. Arnoult, T. Camps, E. Havard, and C. Fontaine, J. Appl. Phys. 101, 024322 (2007).

54 F. Guinea, New J. Phys. 12, 083063 (2010).

55 S. Konschuh, M. Gmitra, D. Kochan, and J. Fabian, Phys.
Rev. B 85, 115423 (2012).

56 E. McCann and M. Koshino, Rep. Prog. Phys. 76, 056503 (2013).

57 A. Kormányos and G. Burkard, Phys. Rev. B 87, 045419 (2013).

58 H. Min, B. Sahu, S. K. Banerjee, and A. H. MacDonald, Phys. Rev. B 75, 155115 (2007).

59 E. V. Castro, K. S. Novoselov, S. V. Morozov, N. M. R. Peres, J. M. B. Lopes dos Santos, J. Nilsson, F. Guinea, A. K. Geim, and A. H. Castro Neto, Phys. Rev. Lett. 99, 216802 (2007).

60 Y. Zhang, T.-T. Tang, C. Girit, Z. Hao, M. C. Martin, A. Zettl, M. F. Crommie, Y. R. Shen, and F. Wang, Nature (London) 459, 820 (2009).

61 J. Nilsson, A. H. Castro Neto, F. Guinea, and N. M. R. Peres, Phys. Rev. B 78, 045405 (2008).

62 E. McCann, arXiv:1205.4849

63 J. K. Viljas and T. T. Heikkilä, Phys. Rev. B 81, 245404 (2010). Its arXiv version arXiv:1002.3502 contains more detailed information.

${ }^{64}$ K. M. Borysenko, J. T. Mullen, X. Li, Y. G. Semenov, J. M. Zavada, M. Buongiorno Nardelli, and K. W. Kim, Phys. Rev. B 83, 161402(R) (2011).

65 E. Cappelluti and G. Profeta, Phys. Rev. B 85, 205436 (2012).

66 T.-Y. Yang, J. Balakrishnan, F. Volmer, A. Avsar, M. Jaiswal, J. Samm, S. R. Ali, A. Pachoud, M. Zeng, M. Popinciuc, G. Güntherodt, B. Beschoten, and B. Özyilmaz, Phys. Rev. Lett. 107, 047206 (2011).

67 M. Diez and G. Burkard, Phys. Rev. B 85, 195412 (2012).

68 I. Neumann, J. Van de Vondel, G. Bridoux, M. V. Costache, F. Alzina, C. M. S. Torres, and S. O. Valenzuela, Small 9, 156 (2013).

69 M. I. D'yakonov and V. I. Perel', Zh. Eksp. Teor. Fiz. 60, 1954 (1971) [Sov. Phys. JETP 33, 1053 (1971)].

70 M. W. Wu, J. H. Jiang, and M. Q. Weng, Phys. Rep. 493, 61 (2010).

71 M. W. Wu and C. Z. Ning, Eur. Phys. J. B 18, 373 (2000); M. W. Wu, J. Phys. Soc. Jpn. 70, 2195 (2001).

72 M. Q. Weng and M. W. Wu, Phys. Rev. B 68, 075312 (2003); 70, 195318 (2004).

73 M. M. Glazov and E. L. Ivchenko, Pis'ma Zh. Eksp. Teor. Fiz. 75, 476 (2002); Zh. Eksp. Teor. Fiz. 126, 1465 (2004) [JETP Lett. 75, 403 (2002); JETP 99, 1279 (2004)].

74 J. Zhou, J. L. Cheng, and M. W. Wu, Phys. Rev. B 75, 045305 (2007).

75 W. J. H. Leyland, G. H. John, R. T. Harley, M. M. Glazov, E. L. Ivchenko, D. A. Ritchie, I. Farrer, A. J. Shields, and M. Henini, Phys. Rev. B 75, 165309 (2007).

76 D. Stich, J. Zhou, T. Korn, R. Schulz, D. Schuh, W. Wegscheider, M. W. Wu, and C. Schüller, Phys. Rev. Lett. 98, 176401 (2007); Phys. Rev. B 76, 205301 (2007).

77 L. F. Han, X. H. Zhang, H. Q. Ni, and Z. C. Niu, Physica E 43, 1127 (2011).

${ }^{78}$ G. F. Koster, J. O. Dimmock, R. G. Wheeler, and H. Statz, Properties of the Thirty-two Point Groups (MIT Press, Cambridge, Massachusetts, 1963).

79 J. H. Jiang and M. W. Wu, Phys. Rev. B 79, 125206 (2009).

80 F. Zhang, H. Z. Zheng, Y. Ji, J. Liu, and G. R. Li, Europhys. Lett. 83, 47006 (2008).

81 T. Korn, D. Stich, R. Schulz, D. Schuh, W. Wegscheider, and C. Schüller, Adv. Solid State Phys. 48, 143 (2009).

82 It is noted that when dealing with the delta function in 
the scattering terms, we neglect the anisotropy of the energy spectrum since the effect of the anisotropy on spin relaxation is marginal ${ }^{67}$

83 S. Piscanec, M. Lazzeri, F. Mauri, A. C. Ferrari, and J. Robertson, Phys. Rev. Lett. 93, 185503 (2004).

84 M. Lazzeri, S. Piscanec, F. Mauri, A. C. Ferrari, and J. Robertson, Phys. Rev. Lett. 95, 236802 (2005).

${ }^{85}$ F. Rana, P. A. George, J. H. Strait, J. Dawlaty, S. Shivaraman, M. Chandrashekhar, and M. G. Spencer, Phys. Rev. B 79, 115447 (2009).

86 M. E. Peskin and D. V. Schroeder, An Introduction to Quantum Field Theory (Addison-Wesley, New York, 1995).

87 D. P. DiVincenzo and E. J. Mele, Phys. Rev. B 29, 1685 (1984).

88 G. Dresselhaus, Phys. Rev. 100, 580 (1955).

89 Y. A. Bychkov and E. I. Rashba, J. Phys. C 17, 6039 (1984); Pis'ma Zh. Eksp. Teor. Fiz 39, 66 (1984) [JETP Lett. 39, 78 (1984)].

90 The electron density in the experiment by Han and Kawakami ${ }^{37}$ is obtained from its arXiv version (arXiv:1012.3435).

91 It is noted that due to the complicated energy spectrum of BLG, we employ the single-layer graphene approximation to calculate the spin diffusion coefficient in BLG according to the report by Zhang and $\mathrm{Wu}{ }^{46}$

92 S. Das Sarma, E. H. Hwang, and E. Rossi, Phys. Rev. B
81, 161407(R) (2010).

93 S. Adam and M. D. Stiles, Phys. Rev. B 82, 075423 (2010).

94 G. A. Baker, Jr., Essentials of Padé Approximants (Academic, New York, 1975).

95 G. D. Mahan, Many-Particle Physics (Plenum, New York, 1990).

96 S. Adam, E. H. Hwang, V. M. Galitski, and S. Das Sarma, Proc. Natl. Acad. Sci. U.S.A. 104, 18392 (2007).

97 E. H. Hwang, S. Adam, and S. Das Sarma, Phys. Rev. Lett. 98, 186806 (2007).

98 S. Adam and S. Das Sarma, Solid State Commun. 146, 356 (2008).

99 B. Wunsch, T. Stauber, F. Sols, and F. Guinea, New J. Phys. 8, 318 (2006).

100 X.-F. Wang and T. Chakraborty, Phys. Rev. B 75, 033408 (2007).

101 E. H. Hwang and S. Das Sarma, Phys. Rev. B 75, 205418 (2007).

102 S. Fratini and F. Guinea, Phys. Rev. B 77, 195415 (2008).

103 O. V. Gmayun, Phys. Rev. B 84, 085112 (2011).

104 E. H. Hwang and S. Das Sarma, Phys. Rev. B 77, 115449 (2008).

105 J.-H. Chen, C. Jang, S. Xiao, M. Ishigami, and M. S. Fuhrer, Nat. Nanotechnol. 3, 206 (2008). 